COURSE 5

\title{
QUANTUM THEORY OF GRAVITATION
}

\author{
Martin J.G. VELTMAN
}

Instituut voor Theoretische Fysica,

Rijksuniversiteit, Sorbonnelaan 4, de Uithof, Utrecht 


\section{Contents}

1. Introduction 267

2. Free spin-2 particles 267

3. The massless spin-2 propagator 272

4. Unitarity 273

5. Extension to non-Abelian group 275

6. Finite gauge transformations 276

7. Invariants 278

8. Covariant derivatives 281

9. Riemann tensor 285

10. Local choice of coordinates; Bianchi identities 286

11. The Lagrangian for pure gravitation 288

12. The cosmological term $\quad 291$

13. Scalar particles 293

14. One-loop divergencies $\quad 296$

15. Divergencies with external gravitational field 307

16. Divergencies with quantized gravitational field 308

17. Evaluation of the divergencies 312

18. Equations of motion $\quad 316$

19. Riemann tensor identities 317

$\begin{array}{ll}\text { 20. Fermions and gravitation } & 320\end{array}$

21. Fermion fields 324

22. Further invariants $\quad 325$

References $\quad 326$

R. Balian andJ. Zinn-Justin, eds., LesHouches, Session XXVIII, 1975 - Methodes en theories des champs / Methods in field theory

(C) North-Holland Publishing Company, 1976 


\section{Introduction}

The quantum theory ofgravitation has been discussed in lectures by various authors, notably Feynman in a course given at CalTec in the academic year 1962-1963, and De Witt, at the 1963 Les Houches school. Since then a certain amount of progress has been made, and it seems proper to emphasize the recent developments in this course.

The progress mentioned stems basically from two facts. One, we now understand much better how to quantize a gauge theory, that is a theory with a Lagrangian possessing an invariance with respect to certain local (i.e. spacetime dependent) transformations. Secondly, the dimensional regularization method turns out to be a powerful tool in handling infinities, and since infinities are plentiful in gravitation theory such a tool is indispensable.

In these lectures we will approach the theory ofgravitation from the point ofview ofquantum field theory. This in itselfposes directly a restriction, namely that we abandon from the start things like curved space and Mach's principle. Of course, the theory that emerges is in the classical limit nothing but Einstein's theory; but the interpretation is really quite different. We refer to the book by Weinberg for the classical theory of gravitation developed from this point of view.

Our starting point will be that gravitation is caused by a particle of spin two. The reasons for that have been given at many occasions, and may be summarized as follows:

(i) The gravitational field cannot be described by a vector field such as the e.m. field because then particles and antiparticles would behave differently, contrary to experiment.

(ii) The gravitational field cannot be described by a scalar field. The reason for this is that we know that gravity couples to energy, which is not a scalar quantity.

\section{Free spin-2 particles}

A particle of spin 2 is described by a symmetric two-index tensor field. We must determine the propagator for such a particle, or equivalently, the part of the Lagrangian quadratic in the tensor field. Consider a source emitting such a particle on mass shell. There should be no way in which this particle at rest can decay into a scalar or spin-1 particle of the same mass. The relevant Feynman diagram is 


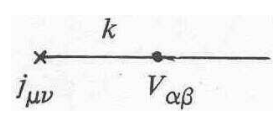

The corresponding expression is

$$
j_{\mu \nu} \frac{f_{\mu \nu \alpha \beta}(k)}{k^{2}+m^{2}-i \epsilon} V_{\alpha \beta},
$$

where $V_{\alpha \beta}$ is the vertex function. In case of decay into a scalar particle it can be proportional to $\delta_{\alpha \beta}$, corresponding to the interaction Lagrangian

$$
\mathcal{L}_{\text {int }}=h_{\mu \mu} \phi,
$$

where $h_{\mu \nu}$ and $\phi$ represent the tensor and the scalar fields respectively. Since (1) must be zero we must have

$$
f_{\mu \nu \alpha \alpha}(k)=0 \text { for } k^{2}=-m^{2} .
$$

Similarly

$$
k_{\beta} f_{\mu \nu \alpha \beta}(k)=0
$$

from the forbiddenness of the interaction

$$
A_{\alpha} \partial_{\beta} h_{\alpha \beta},
$$

where $A_{\alpha}$ is a vector field. Finally, from the fact that the imaginary part of the diagram

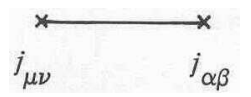

must be positive definite (since by unitarity it is equal to the probability of emitting a spin-2 particle) we find

$$
\begin{aligned}
& f_{\mu \nu \alpha \beta}(k)=c\left[\frac{1}{2}\left(\delta_{\mu \alpha} \delta_{\nu \beta}+\delta_{\mu \beta} \delta_{\nu \alpha}-\delta_{\mu \nu} \delta_{\alpha \beta}\right)\right. \\
& +\frac{1}{2}\left(\delta_{\mu \alpha} \frac{k_{\nu} k_{\beta}}{m^{2}}+\delta_{\nu \beta} \frac{k_{\mu} k_{\alpha}}{m^{2}}+\delta_{\mu \beta} \frac{k_{\nu} k_{\alpha}}{m^{2}}+\delta_{\nu \alpha} \frac{k_{\mu} k_{\beta}}{m^{2}}\right) \\
& \left.+\frac{2}{3}\left(\frac{1}{2} \delta_{\mu \nu}-\frac{k_{\mu} k_{\nu}}{m^{2}}\right)\left(\frac{1}{2} \delta_{\alpha \beta}-\frac{k_{\alpha} k_{\beta}}{m^{2}}\right)\right] .
\end{aligned}
$$


We may perhaps mention that the requirement of positive defmiteness is

$$
z_{\mu \nu} f_{\mu \nu \alpha \beta} z_{\alpha \beta}^{*} \geqslant 0
$$

for any $z$. The quantity $z_{\mathrm{aj} 3}$ is defined as the complex conjugate of $\mathrm{z}$, disregarding the $i$ of the fourth component. Explicitly,

$$
z_{\alpha \beta}^{*}=\left(z_{\alpha \beta}\right)^{*}(-1)^{\delta} \alpha 4(-1)^{\delta}{ }_{\beta 4}
$$

In the restframe where $k=0, £_{4}=i m$ the expression for/Mira $\mathrm{g}$ becomes very simply

$$
f_{\mu \nu \alpha \beta}= \begin{cases}\frac{1}{2}\left(\delta_{\mu \alpha} \delta_{\nu \beta}+\delta_{\mu \beta} \delta_{\nu \alpha}\right)-\frac{1}{3} \delta_{\mu \nu} \delta_{\alpha \beta}, & \text { if } \mu \nu \alpha \beta \neq 4 \\ 0, & \text { otherwise. }\end{cases}
$$

The positive constant $\mathrm{c}$ will be determined to be one.

We must now find a set of symmetric polarization tensors $e_{\mu \nu}^{\lambda}$ such that

$$
\sum_{\lambda} e_{\mu \nu}^{\lambda}(k) e_{\alpha \beta}^{\lambda}(k)=f_{\mu \nu \alpha \beta}(k)
$$

There are five independent tensors satisfying

$$
e_{\mu \mu}=0, \quad k_{\mu} e_{\mu \nu}=0 .
$$

In the $k$ restframe they can be written down readily,

$$
\begin{aligned}
& \sqrt{\frac{2}{3}}\left[\begin{array}{rrrr}
\frac{1}{2} & 0 & 0 & 0 \\
0 & \frac{1}{2} & 0 & 0 \\
0 & 0 & -1 & 0 \\
0 & 0 & 0 & 0
\end{array}\right], \sqrt{ } \frac{1}{2}\left[\begin{array}{rrrr}
1 & 0 & 0 & 0 \\
0 & -1 & 0 & 0 \\
0 & 0 & 0 & 0 \\
0 & 0 & 0 & 0
\end{array}\right], \sqrt{ } \frac{1}{2}\left[\begin{array}{llll}
0 & 1 & 0 & 0 \\
1 & 0 & 0 & 0 \\
0 & 0 & 0 & 0 \\
0 & 0 & 0 & 0
\end{array}\right], \\
& \sqrt{\frac{1}{2}}\left(\begin{array}{llll}
0 & 0 & 1 & 0 \\
0 & 0 & 0 & 0 \\
1 & 0 & 0 & 0 \\
0 & 0 & 0 & 0
\end{array}\right], \quad \sqrt{\frac{1}{2}}\left(\begin{array}{llll}
0 & 0 & 0 & 0 \\
0 & 0 & 1 & 0 \\
0 & 1 & 0 & 0 \\
0 & 0 & 0 & 0
\end{array}\right] .
\end{aligned}
$$

These are now normalized to 1 (i.e. $e^{\wedge}{ }_{v} e^{\wedge}=1$ ). Actually we thus find that $f u, v u, v{ }^{\text {must }}$ be equal to 5 , for $k^{2}=-m^{2}$ which fixes $c=1$ in eq. (2). 
The Lagrangian corresponding to the propagator with the nominator of eq. (2) is (apart from an overall factor of 2)

$$
\begin{aligned}
\mathscr{L}=\mathcal{L}_{\text {sym }} & +\frac{1}{4} m^{2}\left(h_{\mu \mu} h_{\nu \nu}-h_{\mu \nu} h_{\mu \nu}\right), \\
\mathcal{L}_{\text {sym }}= & -\frac{1}{2} \partial_{\lambda} h_{\lambda \mu} \partial_{\mu} h_{\nu \nu}+\frac{1}{2} \partial_{\lambda} h_{\lambda \mu} \partial_{\nu} h_{\nu \mu} \\
& -\frac{1}{4} \partial_{\lambda} h_{\mu \nu} \partial_{\lambda} h_{\mu \nu}+\frac{1}{4} \partial_{\lambda} h_{\mu \mu} \partial_{\lambda} h_{\nu \nu} .
\end{aligned}
$$

This follows by working out the inverse of the function $f_{\mu \nu \alpha \beta}$ in eq. (2). We will do such an inversion in greater detail later on.

All this is straightforward and easy. But we are really interested in the massless case, and then things are much more complicated. Evidently, the limit $m \rightarrow 0$ in eq. (2) makes no sense. We can take the limit $m=0$ in the Lagrangian of eq. (3); if we do that we cannot find a propagator, because the inverse of the Fourier transform of this Lagrangian does not exist. Brief: the various requirements that we started from do not allow a solution if $k^{2}=0$.

Thus, if we are to build a theory ofmassless spin-2 particles we must relax our conditions. But we may relax them only insofar that no physically intolerable consequences result. Let us for a moment consider quantum electrodynamics, where the same situation exists. For massive vector particles we have the propagator

$$
\frac{\delta_{\mu \nu}+k_{\mu} k_{\nu} / m^{2}}{k^{2}+m^{2}-i \epsilon} .
$$

This satisfies the various requirements, such as positive definiteness of the numerator. For a massless vector particle such as the photon one often employs the propagator

$$
\frac{\delta_{\mu \nu}}{k^{2}-i \epsilon} \text {. }
$$

The denominator is not positive definite: when multiplying with a "real" fourvector (i.e. a vector with real space components and an imaginary fourth component) we may get a negative result. However, the situation is saved by gauge invariance. By requiring gauge invariant coupling of the photon to the rest of the world the occurrence of negative probabilities is avoided.

Thus we must introduce some kind of gauge invariance. There are a number 
of requirements that may be written down, such as the fact that the gauge transformations must form a group.

In quantum electrodynamics the gauge transformations are defined such that a scalar component in the photon field decouples. One requires invariance for the transformation

$$
A_{\mu} \rightarrow A_{\mu}+\partial_{\mu} \Lambda
$$

with arbitrary space-time dependent $\Lambda$. Similarly we require now invariance with respect to a transformation that amounts to decoupling vector and scalar parts in the tensor field. We will require invariance under the gauge transformation

$$
h_{\mu \nu} \rightarrow h_{\mu \nu}+\partial_{\mu} \eta_{\nu}+\partial_{\nu} \eta_{\mu}
$$

where the $\eta_{\mu}$ are four arbitrary functions. It must be noted that we leave out a term of the form $\delta_{\mu \nu} \Lambda$; such a term could be included and amounts, ultimately, to a scale transformation (Weyl transformation).

Now that we have defined a gauge invariance we can proceed along the lines known for gauge theories. We leave it to the reader to observe that the Lagrangian $\mathcal{L}_{\text {sym }}$ in eq. (3) is invariant (up to a total derivative) for this transformation. Next we must choose a convenient gauge breaking term, for example,

$$
-\frac{1}{2} C_{\mu}^{2}
$$

with

$$
C_{\mu}=\partial_{\nu} h_{\mu \nu}-\frac{1}{2} \partial_{\mu} h_{\nu \nu} .
$$

With this choice of gauge we have

$$
\mathcal{L}=\mathcal{L}_{\text {sym }}-\frac{1}{2} C^{2}=-\frac{1}{4} \partial_{\lambda} h_{\alpha \beta} \partial_{\lambda} h_{\alpha \beta}+\frac{1}{8} \partial_{\lambda} h_{\alpha \alpha} \partial_{\lambda} h_{\beta \beta},
$$

where $\mathcal{L}_{\text {sym }}$ is defined in eq. (3). This may conveniently be written as

$$
\mathcal{L}=-\frac{1}{2} \partial_{\lambda} h_{\alpha \beta} V_{\alpha \beta \mu \nu} \partial_{\lambda} h_{\mu \nu},
$$

with

$$
V_{\alpha \beta \mu \nu}=\frac{1}{2} \delta_{\alpha \mu} \delta_{\beta \nu}-\frac{1}{4} \delta_{\alpha \beta} \delta_{\mu \nu} .
$$




\section{The massless spin-2 propagator}

Let us now compute the propagator corresponding to eq. (6). To this purpose we rename the ten independent components of $h_{\mu \nu}$ into the ten component quantities $\psi_{i}, i=1, \ldots, 10$. The index correspondence is

$$
\begin{array}{rrrrrrrrrrrr}
h & 11 & 22 & 33 & 44 & 12 & 13 & 14 & 23 & 24 & 34 & \\
\psi & 1 & 2 & 3 & 4 & 5 & 6 & 7 & 8 & 9 & 10 . & (9)
\end{array}
$$

In terms of the $\psi$ the Lagrangian (6) becomes

$$
\mathcal{L}=-\frac{1}{2} \sum_{i=5}^{10} \partial_{\mu} \psi^{i} \partial_{\mu} \psi^{i}-\frac{1}{4} \sum_{i=1}^{4} \partial_{\mu} \psi^{i} \partial_{\mu} \psi^{i}+\frac{1}{8}\left(\partial_{\mu} \sum_{i=1}^{4} \psi^{i}\right)^{2} .
$$

This can be written as

$$
\mathcal{L}=-\frac{1}{2} \partial_{\mu} \psi^{i} V^{i j} \partial_{\mu} \psi^{j},
$$

with

$$
\begin{aligned}
V^{i j} & =\delta^{i j} \quad \text { if } \quad i, j \geqslant 5 \\
& =\left[\begin{array}{rrrr}
\frac{1}{4} & -\frac{1}{4} & -\frac{1}{4} & -\frac{1}{4} \\
-\frac{1}{4} & \frac{1}{4} & -\frac{1}{4} & -\frac{1}{4} \\
-\frac{1}{4} & -\frac{1}{4} & \frac{1}{4} & -\frac{1}{4} \\
-\frac{1}{4} & -\frac{1}{4} & -\frac{1}{4} & \frac{1}{4}
\end{array}\right] \quad \text { if } \quad 1 \leqslant i, j \leqslant 4 \\
& =0 \quad \text { otherwise. }
\end{aligned}
$$

The inverse of this $10 \mathrm{X} 10$ matrix $V$ is

$$
\begin{aligned}
\left(V^{-1}\right)^{i j} & =\delta^{i j} \quad \text { if } i, j \geqslant 5 \\
& =\left[\begin{array}{rrrr}
1 & -1 & -1 & -1 \\
-1 & 1 & -1 & -1 \\
-1 & -1 & 1 & -1 \\
-1 & -1 & -1 & 1
\end{array}\right] \quad \text { if } \quad 1 \leqslant i, j \leqslant 4 \\
& =0 \text { otherwise. }
\end{aligned}
$$


This coincides with the matrix obtained from the expression

$$
\delta_{\mu \alpha} \delta_{\nu \beta}+\delta_{\mu \beta} \delta_{\nu \alpha}-\delta_{\mu \nu} \delta_{\alpha \beta}
$$

if we employ the same index correspondence for the pairs $\mu \nu \rightarrow i$ and $\alpha \beta \rightarrow j$ as given in eq. (9). The propagator corresponding to the Lagrangian (6) is therefore

$$
\frac{\delta_{\mu \alpha} \delta_{\nu \beta}+\delta_{\mu \beta} \delta_{\nu \alpha}-\delta_{\mu \nu} \delta_{\alpha \beta}}{k^{2}-i \epsilon} .
$$

It is interesting to note that there is a difference with the propagator for the massive case even if $k_{\mu}$ is set to zero.

In spaces of dimensionality $n$ instead of 4 as we have here the same procedure leads to the propagator

$$
\frac{1}{k^{2}-i \epsilon}\left\{\delta_{\mu \alpha} \delta_{\nu \beta}+\delta_{\mu \beta} \delta_{\nu \alpha}-\frac{2}{n-2} \delta_{\mu \nu} \delta_{\alpha \beta}\right\} \text {. }
$$

Calculations are somewhat simplified if we introduce the unit matrix $\mathbb{I}^{i j}$.It is given by

$$
\mathbb{1}_{\alpha \beta, \mu \nu}=\frac{1}{2}\left(\delta_{\alpha \mu} \delta_{\nu \beta}+\delta_{\alpha \nu} \delta_{\mu \beta}\right),
$$

with again $\alpha \beta \rightarrow i, \mu \nu \rightarrow j$ according to (9). Notice that

$$
\mathbb{1}_{\alpha \beta, \mu \nu} \mathbb{1}_{\mu \nu, \lambda \kappa}=\mathbb{1}_{\alpha \beta, \lambda \kappa},
$$

also in $n$-dimensional space, where the rule $\delta_{\mu \mu}=n$ holds. Note furthermore that the product of $(8)$ and the expression in brackets in eq. (11) is precisely the unit matrix given in eq. (12) also in $n$-dimensional space.

\section{Unitarity}

An urgent question is to what kind of physics the above procedure corresponds. To this purpose we choose another gauge, the so-called Prentki gauge. We take

$$
C_{\mu}=b \sum_{i=1}^{3} \partial_{i} h_{i \mu}, \quad b \rightarrow \infty .
$$




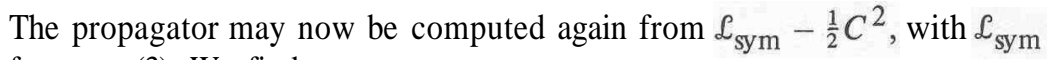
from eq. (3). We find

$$
\begin{aligned}
P_{\mu \nu, \alpha \beta}(k)= & \frac{1}{k^{2}}\left(\bar{\delta}_{\mu \alpha} \bar{\delta}_{\nu \beta}+\bar{\delta}_{\mu \beta} \bar{\delta}_{\nu \alpha}-\frac{2}{n-2} \bar{\delta}_{\mu \nu} \bar{\delta}_{\alpha \beta}\right) \\
& +\frac{1}{k^{2}}\left(\bar{\delta}_{\mu \alpha} \delta_{\nu \beta 4}+\bar{\delta}_{\mu \beta} \delta_{\nu \alpha 4}+\bar{\delta}_{\nu \alpha} \delta_{\mu \beta 4}+\bar{\delta}_{\nu \beta} \delta_{\mu \alpha 4}\right. \\
& \left.-\frac{2}{n-2} \bar{\delta}_{\mu \nu} \delta_{\alpha \beta 4}-\frac{2}{n-2} \bar{\delta}_{\alpha \beta} \delta_{\mu \nu 4}\right)+\frac{2 n-6}{n-2} \frac{k^{2}}{k^{2}} \delta_{\mu \nu \alpha \beta 4},
\end{aligned}
$$

with

$$
\begin{array}{ll}
k^{2}=k^{2}-k_{4}^{2}, & \bar{\delta}_{\mu \nu}=\left(1-\delta_{\mu 4}\right)\left(1-\delta_{\nu 4}\right)\left(\delta_{\mu \nu}-\frac{k_{\mu} k_{\nu}}{k^{2}}\right), \\
\delta_{\mu \nu 4}=\delta_{\mu 4} \delta_{\nu 4}, & \delta_{\mu \nu \alpha \beta 4}=\delta_{\mu 4} \delta_{\nu 4} \delta_{\alpha 4} \delta_{\beta 4} .
\end{array}
$$

In the frame where $k_{1}$ and $k_{2}$ are zero we have, at the pole $k^{2}=0$, that

$k_{3}=|k|$ and $k_{4}=i|k|$. Then $\delta_{\mu \nu}$ is non-zero only for $\mu=\nu=1$ or $\mu=\nu=2$. In four dimensions the residue at the pole $k^{2}=0$ (note: not $\left.k^{2}=0\right)$ is then given by

$$
\begin{aligned}
\text { Residue } & =\delta_{\mu \alpha} \delta_{\nu \beta}+\delta_{\mu \beta} \delta_{\nu \alpha}-\delta_{\mu \nu} \delta_{\alpha \beta} \text { if } \quad \mu, \nu, \alpha, \beta=1,2 \\
& =0 \quad \text { otherwise. }
\end{aligned}
$$

Consider now the two polarization tensors

$$
e_{\mu \nu}^{1}=\sqrt{ } \frac{1}{2}\left(\begin{array}{rrrr}
1 & 0 & 0 & 0 \\
0 & -1 & 0 & 0 \\
0 & 0 & 0 & 0 \\
0 & 0 & 0 & 0
\end{array}\right), \quad e_{\mu \nu}^{2}=\sqrt{ } \frac{1}{2}\left(\begin{array}{llll}
0 & 1 & 0 & 0 \\
1 & 0 & 0 & 0 \\
0 & 0 & 0 & 0 \\
0 & 0 & 0 & 0
\end{array}\right),
$$

The expression(15) is precisely equal to

$$
2 \sum_{\lambda=1}^{2} e_{\mu \nu}^{\lambda} e_{\alpha \beta}^{\lambda} \text {. }
$$


Apart from the factor of 2 we see that the propagator corresponds to the propagation of two polarization modes. The massless graviton has two spin states, as we indeed know to be the correct situation. Note that these polarization tensors are traceless.

We must now comment on the factor of 2 . The situation can be cured if we replace $h$ by $\sqrt{2} h$ in the Lagrangian. This has no effect on the $S$-matrix computed with the help of the Feynman rules. Unitarity however requires that the polarization tensors employed are such that

$$
e_{\mu \nu}^{\lambda} f_{\mu \nu \alpha \beta} e_{\alpha \beta}^{\lambda^{\prime}}=\delta_{\lambda \lambda^{\prime}},
$$

where $f_{\mu \nu \alpha \beta}$ is the residue on-mass-shell of the propagator as given in eq. (15). Using polarization tensors given by $\sqrt{ } \frac{1}{2}$ times those shown in eq. (16) leads to the correct answers.

\section{Extension to non-Abelian group}

Rather than using the gauge invariance under the transformations (4) we will consider a more general possibility including a free parameter $\kappa$. We will require invariance under the replacement

$$
h_{\mu \nu} \rightarrow h_{\mu \nu}+\kappa\left(h_{\alpha \nu} \partial_{\mu} \eta_{\alpha}+h_{\alpha \mu} \partial_{\nu} \eta_{\alpha}+\eta_{\alpha} \partial_{\alpha} h_{\mu \nu}\right)+\partial_{\mu} \eta_{\nu}+\partial_{\nu} \eta_{\mu},
$$

with infinitesimal $\eta$. These gauge transformations are acceptable if they form a group. Since we work with infinitesimal $\eta$ the transformation shown differs by terms of order $\eta^{2}$ and higher from the full gauge transformation. Let us apply first a transformation with a function $\eta$ and next with a function $\bar{\eta}$. Writing only the terms containing both $\eta$ and $\bar{\eta}$ we have

$$
\begin{aligned}
h_{\mu \nu} & \rightarrow h_{\mu \nu}+\kappa\left(\partial_{\alpha} \bar{\eta}_{\nu} \partial_{\mu} \eta_{\alpha}+\partial_{\nu} \bar{\eta}_{\alpha} \partial_{\mu} \eta_{\alpha}+\partial_{\alpha} \bar{\eta}_{\mu} \partial_{\nu} \eta_{\alpha}\right. \\
& \left.+\partial_{\mu} \bar{\eta}_{\alpha} \partial_{\nu} \eta_{\alpha}+\eta_{\alpha} \partial_{\alpha} \partial_{\mu} \bar{\eta}_{\nu}+\eta_{\alpha} \partial_{\alpha} \partial_{\nu} \bar{\eta}_{\mu}\right)+\kappa^{2}\left\{h_{\beta \nu} \partial_{\alpha} \bar{\eta}_{\beta} \partial_{\mu} \eta_{\alpha}\right. \\
& +h_{\beta \alpha} \partial_{\nu} \bar{\eta}_{\beta} \partial_{\mu} \eta_{\alpha}+\bar{\eta}_{\beta} \partial_{\beta} h_{\alpha \nu} \partial_{\mu} \eta_{\alpha}+h_{\beta \mu} \partial_{\alpha} \bar{\eta}_{\beta} \partial_{\nu} \eta_{\alpha}+h_{\beta \alpha} \partial_{\mu} \bar{\eta}_{\beta} \partial_{\nu} \eta_{\alpha} \\
& \left.+\bar{\eta}_{\beta} \partial_{\beta} h_{\alpha \mu} \partial_{\nu} \eta_{\alpha}+\eta_{\beta} \partial_{\beta}\left(h_{\alpha \nu} \partial_{\mu} \bar{\eta}_{\alpha}+h_{\alpha \mu} \partial_{\nu} \bar{\eta}_{\alpha}+\bar{\eta}_{\alpha} \partial_{\alpha} h_{\mu \nu}\right)\right\} \\
& + \text { terms linear in } \eta \text { or } \bar{\eta}+\mathrm{O}\left(\eta^{2}, \bar{\eta}^{2}\right) .
\end{aligned}
$$


This must be equal to a gauge transformation with a function $\overline{\bar{\eta}}$ given by

$$
\bar{\eta}_{\alpha}=\eta_{\alpha}+\bar{\eta}_{\alpha}+\frac{1}{2} c_{\alpha \beta \gamma} \eta_{\beta} \bar{\eta}_{\gamma}+\ldots,
$$

with structure constants $c_{\alpha \beta \gamma}$. Terms containing $\eta$ and $\bar{\eta}$ arise from lowest order $\overline{\bar{\eta}}$, or from terms $\bar{\eta}^{2}$ in the gauge transformations, of which we do not know the explicit form. However, the latter are symmetric in $\eta$ and $\bar{\eta}$, and if we restrict ourselves to terms antisymmetric in $\eta$ and $\bar{\eta}$ they will not bother us. We leave it to the reader to check that the gauge transformation (18) antisymmetrized in $\eta$ and $\bar{\eta}$ equalsagaugetransformationoftheform(17), with instead of $\eta$ the function

$$
\frac{1}{2} \kappa\left(\eta_{\beta} \partial_{\beta} \bar{\eta}_{\alpha}-\bar{\eta}_{\beta} \partial_{\beta} \eta_{\alpha}\right) .
$$

This also shows that we are dealing with structure constants containing derivatives.

\section{Finite gauge transformations}

Consider the functions $\xi_{p}(x)$ and $\bar{\xi}_{p}(x)$. Clearly a function of a function of $x$ is again a function of $x$,

$$
\bar{\xi}_{\nu}(x) \equiv \bar{\xi}_{\nu}\left(\xi_{\mu}(x)\right) \text {. }
$$

Let now $\xi$ and $\bar{\xi}$ be infinitesimally near to the unit function

$$
\xi_{\nu}(x)=x_{\nu}+\eta_{\nu}(x)+\ldots, \quad \bar{\xi}_{\nu}(x)=x_{\nu}+\bar{\eta}_{\nu}(x)+\ldots .
$$

We find

$$
\begin{aligned}
\overline{\bar{\xi}}_{\nu}(x) & =\xi_{\nu}(x)+\bar{\eta}_{\nu}\left(\xi_{\nu}(x)\right) \\
& =x_{\nu}+\eta_{\nu}(x)+\bar{\eta}_{\nu}(x)+\frac{\partial \bar{\eta}_{\nu}(x)}{\partial x_{\alpha}} \eta_{\alpha}(x)+\ldots .
\end{aligned}
$$

If we write

$$
\overline{\bar{\xi}}_{\nu}(x)=x_{\nu}+\overline{\bar{\eta}}_{\nu}(x),
$$


we have

$$
\bar{\eta}_{\nu}(x)=\eta_{\nu}(x)+\bar{\eta}_{\nu}(x)+\eta_{\alpha} \frac{\partial \bar{\eta}_{\nu}}{\partial x_{\alpha}}+\ldots .
$$

Antisymmetrizing this gives us indeed the result of eq. (20).

The finite form of which eq. (17) is the infinitesimal part is easily obtained. We may rewrite

$$
h_{\mu \nu} \rightarrow h_{\mu \nu}+g_{\alpha \nu} \partial_{\mu} \eta_{\alpha}+g_{\alpha \mu} \partial_{\nu} \eta_{\alpha}+\eta_{\alpha} \partial_{\alpha} g_{\mu \nu},
$$

where

$$
g_{\mu \nu} \equiv \delta_{\mu \nu}+\kappa h_{\mu \nu} .
$$

Eliminating $h$ altogether we find from eq. (25)

$$
g_{\mu \nu} \rightarrow g_{\mu \nu}+\kappa g_{\alpha \nu} \partial_{\mu} \eta_{\alpha}+\kappa g_{\alpha \mu} \partial_{\nu} \eta_{\alpha}+\kappa \eta_{\alpha} \partial_{\alpha} g_{\mu \nu} .
$$

This is the infinitesimal form of

$$
g_{\mu \nu} \rightarrow X_{\alpha \mu} X_{\beta \mu} g_{\alpha \beta}(\xi(x)),
$$

with

$$
\begin{aligned}
& X_{\alpha \mu}=\frac{\partial \xi_{\alpha}(x)}{\partial x_{\mu}}, \\
& \xi_{\alpha}(x)=x_{\alpha}+\kappa \eta_{\alpha}(x)+\ldots .
\end{aligned}
$$

As a matter of convenience we will from now on write the index of the $\eta$ on top. Thus eq. (27) becomes

$$
g_{\mu \nu} \rightarrow g_{\mu \nu}+\kappa g_{\alpha \nu} \partial_{\mu} \eta^{\alpha}+\kappa g_{\alpha \mu} \partial_{\nu} \eta^{\alpha}+\kappa \eta^{\alpha} \partial_{\alpha} g_{\mu \nu} .
$$

The inverse of the tensor $g_{\mu \nu}$ will be denoted by $g^{\mu \nu}$;it is an infinite series in terms of $h_{\mu \nu}$. Wehave

$$
g^{\mu \nu} \equiv \delta^{\mu \nu}+\kappa h^{\mu \nu}, \quad h^{\mu \nu} \equiv-h_{\mu \nu}+\kappa h_{\mu \alpha} h_{\alpha \nu}-\kappa^{2} h_{\mu \alpha} h_{\alpha \beta} h_{\beta \nu}+\ldots
$$


The transformation properties of $g^{\mu \nu}$ follow from those of $g_{\mu \nu}$,

$$
g^{\mu \nu}(x) \rightarrow g^{\alpha \beta}(\xi(x)) \frac{\partial x^{\mu}}{\partial \xi^{\alpha}(x)} \frac{\partial x^{\nu}}{\partial \xi^{\beta}(x)} .
$$

Note that we must in fact use the transposed of the inverse of the matrix $X$ defined above. In the infinitesimal form that leads to an exchange ofindices on top of the minus sign. The infinitesimal form of the transformation is

$$
g^{\mu \nu} \rightarrow g^{\mu \nu}-\kappa g^{\alpha \nu} \partial_{\alpha} \eta^{\mu}-\kappa g^{\mu \alpha} \partial_{\alpha} \eta^{\nu}+\kappa \eta^{\alpha} \partial_{\alpha} g^{\mu \nu} .
$$

The last term arises from the expansion of $g^{\alpha \beta}(x+\kappa \eta)$.

It is interesting to note that if we define $h^{\mu \nu}$ as in eq. (31) we would have obtained another gauge transformation satisfying the group requirement and equal to the starting point eq. (4) (with $-\eta$ instead of $\eta$ )

$$
h^{\mu \nu} \rightarrow h^{\mu \nu}-\kappa\left(h^{\alpha \alpha} \partial_{\alpha} \eta^{\mu}+h^{\mu \alpha} \partial_{\alpha} \eta^{\nu}-\eta^{\alpha} \partial_{\alpha} h^{\mu \nu}\right)-\partial^{\nu} \eta^{\mu}-\partial^{\mu} \eta^{\nu} .
$$

We leave it to the reader to check this last transformation against the result obtained from eq. (31) using the transformation properties of $h_{\mu \nu}$.

\section{Invariants}

We have already one invariant in the theory, namely

$$
g^{\mu \alpha} g_{\alpha \nu} \equiv \delta_{\nu}^{\mu} .
$$

If we consider eq. (28) then we see that matrices $X_{\alpha \mu}$ are arbitrary $4 \mathrm{X} 4$ matrices without special properties except their reality properties. Their determinant may be anything, except that we exclude determinant zero. The only invariants that can be build up are then obviously quantities that transform partly by $X$, partly by $X^{-1}$. That indeed is the case with $g_{\mu \nu}$ and $g^{\mu \nu}$. It is now easy to extend the procedure to include scalars, vectors, etc.

We must not forget what is the goal of our investigation. The idea is to build up a gauge invariant Lagrangian (it may change only by a total derivative) giving the interaction of the gravitational field with itself and other fields. Thus it is necessary to invent transformation properties for the other fields such that gauge invariant expressions can be build up. It will not be possible to work with invariant fields if we require the existence of one-graviton ex- 
change diagrams. And we really need those diagrams because they contain Newton's law. Only one-graviton exchange can give $1 / r$ behaviour. Now, this requires a vertex of the form

$$
h_{\mu \nu} T_{\mu \nu} \text { (matter) }
$$

containing only once the gravitational field. In here $T_{\mu \nu}$ contains the other fields, in particular there must be a quadratic term for every elementary particle.

Evidently, invariance of eq. (36) with invariant $T_{\mu \nu}$ is not possible unless $k$ is taken zero in the transformation law (17). In that case we have

$$
\begin{aligned}
& h_{\mu \nu} T_{\mu \nu} \rightarrow h_{\mu \nu} T_{\mu \nu}+\left(\partial_{\mu} \eta_{\nu}+\partial_{\nu} \eta_{\mu}\right) T_{\mu \nu} \\
& \quad=h_{\mu \nu} T_{\mu \nu}-\eta_{\nu} \partial_{\mu} T_{\mu \nu}-\eta_{\mu} \partial_{\nu} T_{\mu \nu}+\text { total derivative. }
\end{aligned}
$$

If $T$ is divergence free this is invariant (apart from the irrelevant total derivative). However, to make a divergence free $T_{\mu \nu}$ is very difficult, because one is not allowed to use equations ofmotion in the Lagrangian. For instance, the traditional $T_{\mu \nu}$ for a scalar field

$$
T_{\mu \nu}=\partial_{\mu} \phi \partial_{\nu} \phi-m^{2} \phi^{2} \delta_{\mu \nu}
$$

is only divergence free because of the equation of motion

$$
\left(\partial^{2}-m^{2}\right) \phi=0 \text {. }
$$

Transformation properties for the fields must be invented and assigned.

Following the discussion above, and considering the transformation (28) of $g_{\mu \nu}$ we can write down transformation properties for vectors such that invariants result if these vectors are contracted with $g_{\mu \nu}$.

Let there be given a vector field $A_{\mu}(x)$. We assign the transformation law

$$
A_{\mu}(x) \rightarrow X_{\alpha \mu} A_{\alpha}(\xi(x))
$$

with $X$ from eq. (29). The infinitesimal form is

$$
A_{\mu} \rightarrow A_{\mu}+A_{\alpha} \partial_{\mu} \eta^{\alpha}+\eta^{\alpha} \partial_{\alpha} A_{\mu} .
$$

Here $A_{\mu}$ is called a covariant vector. Let $A^{\mu}$ be defined by 


$$
A^{\mu} \equiv g^{\mu \nu} A_{\nu}
$$

We find for the transformation property of this $A^{\mu}$ as a consequence of the transformation properties of $g^{\mu \nu}$ and $A_{\nu}$,

$$
A^{\mu} \rightarrow A^{\mu}-A^{\alpha} \partial_{\alpha} \eta^{\mu}+\eta^{\alpha} \partial_{\alpha} A^{\mu} .
$$

Obviously $A^{\mu}$ transforms with $\left(X^{-1}\right)^{T}$. $A^{\mu}$ will be called a contravariant vector.

By construction, it is clear that in transforming the quantity $g^{\mu \nu} A_{\mu} A_{\nu}$ the matrix $X$ will cancel out. However, it is still not an invariant,

$$
g^{\mu \nu}(x) A_{\mu}(x) A_{\nu}(x) \rightarrow g^{\mu \nu}(\xi(x)) A_{\mu}(\xi(x)) A_{\nu}(\xi(x)) .
$$

The infinitesimal form of this is

$$
g^{\mu \nu} A_{\mu} A_{\nu} \rightarrow g^{\mu \nu} A_{\mu} A_{\nu}+\kappa \eta^{\alpha} \partial_{\alpha}\left(g^{\mu \nu} A_{\mu} A_{\nu}\right) .
$$

A field $\phi(x)$ is therefore called a scalar field if the assigned transformation law is

$$
\phi \rightarrow \phi+\kappa \eta^{\alpha} \partial_{\alpha} \phi .
$$

We have still no invariant. How can we construct something that changes at most by a total derivative? Let us consider the transformation properties of the determinant of $g_{\mu \nu}$. We rewrite first the transformation law (30) for the $g_{\mu \nu}$

$$
g_{\mu \nu} \rightarrow g_{\mu \beta}\left[\delta_{\nu}^{\beta}+\left(g^{\beta \gamma} g_{\alpha \nu} \partial_{\gamma} \eta^{\alpha}+g^{\beta \gamma} g_{\gamma \alpha} \partial_{\nu} \eta^{\alpha}+\eta^{\alpha} g^{\beta \gamma} \partial_{\alpha} g_{\gamma \nu}\right)\right],
$$

where we also absorbed $\kappa$ into $\eta$. In brackets we have the unit matrix plus something of order $\eta$. As is well known

$$
\operatorname{det}(1+A)=1+\operatorname{Tr}(A)+\mathrm{O}\left(A^{2}\right),
$$

where $\operatorname{Tr}$ stands for trace. We so find

$$
\begin{aligned}
\operatorname{det} & g \rightarrow \operatorname{det} g[1+\operatorname{Tr}()] \\
= & \operatorname{det} g\left[1+2 \partial_{\alpha} \eta^{\alpha}+\eta^{\alpha} g^{\beta \gamma} \partial_{\alpha} g_{\gamma \beta}\right]
\end{aligned}
$$

to first order in $\eta$. 
Next we compute the derivative of det $g$ to an arbitrary power $\lambda$,

$$
\partial_{\alpha}(\operatorname{det} g)^{\lambda}=\lambda(\operatorname{det} g)^{\lambda-1} \partial_{\alpha}(\operatorname{det} g) .
$$

Since

$$
\begin{aligned}
& \begin{aligned}
g_{\mu \nu}(x+\mathrm{d} x) & =g_{\mu \nu}(x)+\mathrm{d} x^{\alpha} \partial_{\alpha} g_{\mu \nu} \\
& =g_{\mu \beta}\left(\delta_{\nu}^{\beta}+g^{\beta \gamma} \partial_{\alpha} g_{\gamma \nu} \mathrm{d} x^{\alpha}\right),
\end{aligned} \\
& \operatorname{det}(g(x+\mathrm{d} x))=\operatorname{det} g\left(1+g^{\beta \gamma} \partial_{\alpha} g_{\gamma \beta} \mathrm{d} x^{\alpha}\right), \\
& \partial_{\alpha}(\operatorname{det} g)=\operatorname{det} g g^{\beta \gamma} \partial_{\alpha} g_{\gamma \beta} .
\end{aligned}
$$

We so find

$$
\partial_{\alpha}\left[(\operatorname{det} g)^{\lambda} \eta^{\alpha}\right]=(\operatorname{det} g)^{\lambda}\left\{\partial_{\alpha} \eta^{\alpha}+\lambda g^{\beta \gamma} \partial_{\alpha} g_{\gamma \beta} \eta^{\alpha}\right\} .
$$

Eqs. (45) and (46) may be identified if $\lambda=\frac{1}{2}$; the transformation of $(\operatorname{det} g)^{1 / 2}$ following from (45) may be rewritten as

$$
(\operatorname{det} g)^{1 / 2} \rightarrow(\operatorname{det} g)^{1 / 2}+\partial_{\alpha}\left\{\eta^{\alpha}(\operatorname{det} g)^{1 / 2}\right\} .
$$

Thus $(\operatorname{det} g)^{1 / 2}$ changes by a total derivative under a gauge transformation. However, there is more. Consider the product of $(\operatorname{det} g)^{1 / 2}$ and a scalar $\phi$ as defined above. As a matter of notation we will write simply $g$ instead of det $g$. The transformation law is

$$
\begin{aligned}
& \sqrt{g} \phi \rightarrow \sqrt{g} \phi+\partial_{\alpha}\left(\eta^{\alpha} \sqrt{g}\right) \phi+\sqrt{g} \eta^{\alpha} \partial_{\alpha} \phi \\
& =\sqrt{g} \phi+\partial_{\alpha}\left(\eta^{\alpha} \sqrt{g} \phi\right) .
\end{aligned}
$$

Thus also the product of $\sqrt{ } g$ with a scalar changes only by a total derivative.

If we now construct a Lagrangian that behaves as a scalar and multiply subsequently by $\sqrt{ } g$ we then have a gauge invariant expression.

\section{Covariant derivatives}

Consider the derivative of a vector field $A_{\mu}$. From eq. (40) we deduce its transformation property, 


$$
\begin{gathered}
\partial_{\nu} A_{\mu} \rightarrow \partial_{\nu} A_{\mu}+\partial_{\nu} A_{\alpha} \partial_{\mu} \eta^{\alpha}+A_{\alpha} \partial_{\nu} \partial_{\mu} \eta^{\alpha} \\
+\partial_{\alpha} A_{\mu} \partial_{\nu} \eta^{\alpha}+\eta^{\alpha} \partial_{\alpha} \partial_{\nu} A_{\mu} .
\end{gathered}
$$

This is almost the transformation law for a tensor (compare eq. (30)) except for the term $\partial_{\nu} \partial_{\mu} \eta^{\alpha}$. We must devise something that eliminates this term. We thus try to invent quantities $\Gamma$ such that

$$
\Gamma_{\nu \mu}^{\beta} A_{\beta} \rightarrow\left(\Gamma_{\nu \mu}^{\beta} A_{\beta}\right)_{\text {tensor }}+A_{\beta} \partial_{\nu} \partial_{\mu} \eta^{\beta} .
$$

The first part shows transformation as a tensor. If we have such $\Gamma$ then

$$
D_{\nu} A_{\mu} \equiv \partial_{\nu} A_{\mu}-\Gamma_{\nu \mu}^{\beta} A_{\beta}
$$

transforms as a tensor.

The problem therefore poses itselfas follows: find quantities $\Gamma$ such that

$$
\Gamma_{\nu \mu}^{\beta} \rightarrow\left(\Gamma_{\nu \mu}^{\beta}\right)_{\text {tensor }}+\partial_{\nu} \partial_{\mu} \eta^{\beta} .
$$

Such a $\Gamma$ cannot be constructed from a vector. But it can be constructed from a two-tensor having an inverse, for which we can take for example $g_{\mu \nu}$, up to now the only two-tensor used. Consider

$$
\Gamma_{\nu \mu}^{\beta} \equiv \frac{1}{2} g^{\beta \alpha}\left(\partial_{\nu} g_{\mu \alpha}+\partial_{\mu} g_{\nu \alpha}-\partial_{\alpha} g_{\mu \nu}\right) .
$$

We find as above,

$$
\partial_{\nu} g_{\mu \alpha} \rightarrow\left(\partial_{\nu} g_{\mu \alpha}\right)_{3 \text {-tensor }}+g_{\beta \alpha}\left(\partial_{\nu} \partial_{\mu} \eta^{\beta}\right)+g_{\mu \beta}\left(\partial_{\nu} \partial_{\alpha} \eta^{\beta}\right) .
$$

From this follows easily the behaviour (52) for the $\Gamma$.

The same quantities $\Gamma$ can be used to construct a covariant derivative of a contravariant vector

$$
D_{\nu} A^{\mu} \equiv \partial_{\nu} A^{\mu}+\Gamma_{\nu \alpha}^{\mu} A^{\alpha}
$$

which behaves as a mixed two-tensor.

In general 


$$
\begin{gathered}
D_{\lambda} T_{\mu \nu \ldots}^{\alpha \beta \ldots} \equiv \partial_{\lambda} T_{\mu \nu \ldots}^{\alpha \beta \ldots}+\Gamma_{\lambda \sigma}^{\alpha} T_{\mu \nu \ldots}^{\sigma \beta \ldots}+\Gamma_{\lambda \sigma}^{\beta} T_{\mu \nu \ldots}^{\alpha \sigma \ldots} \\
-\Gamma_{\mu \lambda}^{\sigma} T_{\sigma \nu \ldots}^{\alpha \beta \ldots}-\Gamma_{\nu \lambda}^{\sigma} T_{\mu \sigma \ldots}^{\alpha \beta \ldots}-\ldots
\end{gathered}
$$

defines a properly behaving tensor.

Some properties may now be quoted:

(i) $\Gamma$ is by construction symmetric in the lower indices

$$
\Gamma_{\mu \nu}^{\alpha}=\Gamma_{\nu \mu}^{\alpha}
$$

As a consequence

$$
D_{\mu} A_{\nu}-D_{\nu} A_{\mu}=\partial_{\mu} A_{\nu}-\partial_{\nu} A_{\mu} .
$$

(ii) The covariant derivative of the tensor used for the construction of $\Gamma$ is zero. Here we have taken $g$; we have

$$
D_{\alpha} g_{\mu \nu}=\partial_{\alpha} g_{\mu \nu}-\Gamma_{\alpha \mu}^{\beta} g_{\beta \nu}-\Gamma_{\alpha \nu}^{\beta} g_{\mu \beta} \text {. }
$$

Use of the defining eq. (53) leads to

$$
D_{\alpha} g_{\mu \nu}=0
$$

(iii) Similarly

$$
D_{\alpha} g^{\mu \nu}=0
$$

(iv) The definition of covariant derivative obeys the chain rule. For example

$$
\begin{aligned}
D_{\alpha}\left(A_{\mu} A^{\nu}\right) & =\partial_{\alpha}\left(A_{\mu} A^{\nu}\right)-\Gamma_{\alpha \mu}^{\beta}\left(A_{\beta} A^{\nu}\right)+\Gamma_{\alpha \beta}^{\nu}\left(A_{\mu} A^{\beta}\right) \\
& =\left(D_{\alpha} A_{\mu}\right) A^{\nu}+A_{\mu}\left(D_{\alpha} A^{\nu}\right)
\end{aligned}
$$

From this, on multiplying with $\delta_{\mu}^{\nu}$,

$$
D_{\alpha}\left(A_{\mu} A^{\mu}\right)=\partial_{\alpha}\left(A_{\mu} A^{\mu}\right),
$$

which is in fact the rule for covariant differentiation of a scalar. Indeed, if $\phi(x)$ is a scalar, then $\partial_{\mu} \phi(x)$ transforms as a vector, 


$$
\partial_{\mu} \phi \rightarrow \partial_{\mu}\left(\phi+\eta^{\alpha} \partial_{\alpha} \phi\right)=\partial_{\mu} \phi+\partial_{\alpha} \phi \partial_{\mu} \eta^{\alpha}+\eta^{\alpha} \partial_{\alpha} \partial_{\mu} \phi .
$$

(v) $\Gamma$ is connected to det $g$. Indeed, from the definition (53) we have

$$
\begin{aligned}
\Gamma_{\mu \beta}^{\beta} & =\frac{1}{2} g^{\beta \alpha} \partial_{\mu} g_{\beta \alpha} \\
& =\frac{1}{\sqrt{g}} \partial_{\mu} \sqrt{g},
\end{aligned}
$$

where we used methods shown before.

(vi) Let $A^{\mu}$ be a contravariant vector. Then

$$
\sqrt{g} D_{\mu} A^{\mu}=\text { total derivative. }
$$

Indeed,

$$
\begin{aligned}
\sqrt{g} D_{\mu} A^{\mu} & =\sqrt{g} \partial_{\mu} A^{\mu}+\sqrt{g} \Gamma_{\mu \alpha}^{\mu} A^{\alpha} \\
& =\sqrt{g} \partial_{\mu} A^{\mu}+\partial_{\alpha} \sqrt{g} A^{\alpha} \\
& =\partial_{\mu}\left(\sqrt{g} A^{\mu}\right) .
\end{aligned}
$$

The properties (iv) and (vi) are very important because they tell us that we can manipulate covariant derivatives in Lagrangiansjust as we were used to. For example,

$$
\begin{aligned}
\sqrt{g} A^{\lambda} D_{\lambda} \phi & =\sqrt{g} D_{\lambda}\left(A^{\lambda} \phi\right)-\sqrt{g}\left(D_{\lambda} A^{\lambda}\right) \phi \\
& =-\sqrt{g}\left(D_{\lambda} A^{\lambda}\right) \phi+\text { total derivative }
\end{aligned}
$$

In this connection it may be mentioned that

$$
\sqrt{g} A^{\mu}
$$

is called a density. From the above we see

$$
\partial_{\mu}\left(\sqrt{g} A^{\mu}\right)=\sqrt{g} D_{\mu} A^{\mu} .
$$




\section{Riemann tensor}

Meanwhile we have come in a strange situation. How can we build a gauge invariant expression for the gravitational field alone? At our disposal is the tensor $g_{\mu \nu}$, and we must construct a scalar.

Unfortunately, covariant derivatives of $g$ are zero. Let us therefore start from the $\Gamma$ as a starting point. The transformation property of $\Gamma$ is very close to that of a tensor (see eq. (52)); it makes sense to consider the covariant derivative of $\Gamma$ and then symmetrize so that the non-covariant pieces cancel.

Thus consider the transformation of the covariant derivative of $\Gamma$,

$$
D_{\alpha} \Gamma_{\sigma \beta}^{\mu} \rightarrow\left(D_{\alpha} \Gamma_{\sigma \beta}^{\mu}\right)_{\text {tensor }}+D_{\alpha} \partial_{\sigma} \partial_{\beta} \eta^{\mu}
$$

Subtracting the same with $\alpha$ and $\beta$ interchanged removes the third derivative of $\eta$. We are left with

$$
-\Gamma_{\alpha \sigma}^{\nu} \partial_{\nu} \partial_{\beta} \eta^{\mu}-\Gamma_{\alpha \beta}^{\nu} \partial_{\sigma} \partial_{\nu} \eta^{\mu}+\Gamma_{\alpha \nu}^{\mu} \partial_{\sigma} \partial_{\beta} \eta^{\nu}-(\alpha \leftrightarrow \beta)
$$

These terms have the precise structure of $\Gamma$ times the anomalous part in the $\Gamma$ transformation and can therefore be cancelled away. Indeed

$$
-\Gamma_{\alpha \sigma}^{\nu} \Gamma_{\nu \beta}^{\mu}+\Gamma_{\alpha \nu}^{\mu} \Gamma_{\sigma \beta}^{\nu}
$$

is already antisymmetric in $\alpha$ and $\beta$, and the anomalous transformation properties are precisely those shown in eq. (68). Thus we now can write down a tensor

$$
\begin{aligned}
R_{\sigma \alpha \beta}^{\mu} & \equiv D_{\alpha} \Gamma_{\sigma \beta}^{\mu}-D_{\beta} \Gamma_{\sigma \alpha}^{\mu}+\Gamma_{\alpha \sigma}^{\nu} \Gamma_{\nu \beta}^{\mu}-\Gamma_{\alpha \nu}^{\mu} \Gamma_{\sigma \beta}^{\nu} \\
& =\partial_{\alpha} \Gamma_{\sigma \beta}^{\mu}-\partial_{\beta} \Gamma_{\sigma \alpha}^{\mu}+\Gamma_{\nu \alpha}^{\mu} \Gamma_{\sigma \beta}^{\nu}-\Gamma_{\nu \beta}^{\mu} \Gamma_{\sigma \alpha}^{\nu}
\end{aligned}
$$

This tensor is called the Riemann tensor. It is antisymmetric in $\alpha$ and $\beta$. By multiplication with $g_{\nu \mu}$ one obtains a tensor with only lower indices,

$$
R_{\nu \sigma \alpha \beta} \equiv g_{\nu \mu} R_{\sigma \alpha \beta}^{\mu} .
$$

It is antisymmetric in $\nu \sigma$ as well. Furthermore it is symmetric for the interchange $\nu \sigma \rightarrow \alpha \beta$,

$$
R_{\nu \sigma \alpha \beta}=R_{\alpha \beta \nu \sigma} \text {. }
$$


Finally it obeys a cyclic symmetry in the last three indices

$$
R_{\mu \nu \alpha \beta}+R_{\mu \beta \nu \alpha}+R_{\mu \alpha \beta \nu}=0 .
$$

By multiplication with $g$ a two-tensor and a scalar may be defined,

$$
R_{\mu \nu} \equiv g^{\alpha \beta} R_{\alpha \mu \nu \beta}, \quad R \equiv g^{\mu \nu} R_{\mu \nu} .
$$

Note

$$
R_{\mu \nu}=R_{\nu \mu} .
$$

\section{Local choice of coordinates; Bianchi identities}

In special relativity many equations can be proven easily by going to a particular coordinate system. Here that is also the case. One writes an equation invariant under gauge transformations and then goes to a particular gauge. Actually, for a given point $\underline{x}$ there exists a gauge such that

$$
\begin{array}{ll}
h_{\mu \nu}(x)=0 & \text { for all } \mu, \nu, \\
\partial_{\alpha} h_{\mu \nu}(x)=0 & \text { for all } \alpha, \mu, \nu .
\end{array}
$$

To see this we write an infinitesimal gauge transformation

$$
h_{\mu \nu} \rightarrow h_{\mu \nu}+\partial_{\nu} \eta^{\mu}+\partial_{\mu} \eta^{\nu} .
$$

The question is: can $\eta$ be chosen such that the right-hand side is zero in a particular point $x$ for any $h$ (obviously taken to be infinitesimal here). Similarly for the derivative of this expression.

Now develop $h$ and $\eta$ around this point $\underline{x}$. With $x=\underline{x}+y$ we have

$$
\begin{aligned}
& h_{\mu \nu}(x)=b_{\mu \nu}+b_{\mu \nu \alpha} y_{\alpha}+b_{\mu \nu \alpha \beta} y_{\alpha} y_{\beta}+\ldots, \\
& \eta^{\mu}(x)=a_{\mu}+a_{\mu \alpha} y_{\alpha}+a_{\mu \alpha \beta} y_{\alpha} y_{\beta}+a_{\mu \alpha \beta \gamma} y_{\alpha} y_{\beta} y_{\gamma}+\ldots .
\end{aligned}
$$

Setting the right-hand side of $(77)$ to zero leads to equations for the unknown coefficients $a$ in terms of the $b$. In the point $x=\underline{x}$ we must have 


$$
b_{\mu \nu}+2 a_{\mu \nu}=0 .
$$

Evidently, for given $b_{\mu \nu}$ we can find $a_{\mu \nu}$ such that this holds. For the first derivative we have

$$
\partial_{\alpha} h_{\mu \nu}+\partial_{\alpha} \partial_{\nu} \eta^{\mu}+\partial_{\alpha} \partial_{\mu} \eta^{\nu}=0
$$

or, in the point $x=\underline{x}$,

$$
b_{\mu \nu \alpha}+2 a_{\mu \nu \alpha}+2 a_{\nu \mu \alpha}=0 \text {. }
$$

This is more tricky because $b$ is symmetrical in the first two indices, and the $a$ in the last two. Nevertheless, this can, for given $b$, be solved for the $a$. Let $\mu, \nu, \alpha$ take some particular value, say $\mu=1, v=2$ and $\alpha=3$. There are three independent $b$ and three independent $a$. We must solve

$$
\begin{aligned}
& -b_{123}=2 a_{123}+2 a_{213}, \\
& -b_{132}=2 a_{123}+2 a_{312}, \\
& -b_{231}=2 a_{213}+2 a_{312} .
\end{aligned}
$$

This set of equations can be solved,

$$
4 a_{213}=-b_{123}+b_{132}-b_{231},
$$

or, generally,

$$
a_{\mu \nu \alpha}=\frac{1}{4}\left(-b_{\nu \mu \alpha}+b_{\nu \alpha \mu}-b_{\mu \alpha \nu}\right) .
$$

Thus an infinitesimal gauge transformation may be chosen such that an infinitesimal $h_{\mu \nu}$ and its first derivatives are eliminated in some point. Repeated application may be used to remove finite $h$ and its derivatives in that point.

Now choose some point $\underline{x}$. The above shows that by a gauge transformation one can arrive at a $g$ such that

$$
g_{\mu \nu}(x)=\delta_{\mu \nu}, \quad \partial_{\alpha} g_{\mu \nu}(x)=0 .
$$

Obviously similar equations hold for $g^{\mu \nu}(x)$. In that point we then have also vanishing $\Gamma$, but the derivatives of $\Gamma$ in general cannot be made zero. Further- 
more, in that point $D_{\mu}=\partial_{\mu}$. We leave it to the reader to check that in the point $\underline{x}$ we have the identity

$$
D_{\gamma} R_{\beta \mu \nu}^{\alpha}+D_{\nu} R_{\beta \gamma \mu}^{\alpha}+D_{\mu} R_{\beta \nu \gamma}^{\alpha}=0 .
$$

In fact, in the point $\underline{x}$ this equation reduces to

$$
-\frac{1}{2} g^{\alpha \lambda}\left[\partial_{\nu} \partial_{\gamma}\left(\partial_{\mu} g_{\beta \lambda}+\partial_{\beta} g_{\mu \lambda}-\partial_{\lambda} g_{\beta \mu}\right)-\mu \leftrightarrow \nu\right]+\operatorname{cycl} .(\gamma \mu \nu)=0 .
$$

Since eq. (80) is gauge invariant it holds also before gauge transforming and is therefore generally true. The eqs. (80) are called Bianchi identities. Note that the tensor $R$ depends on second derivatives and can in general not be made zero by a gauge transformation. In the expansions (78) there are 100 independent coefficients $b_{\mu \nu \alpha \beta}$ and 80 independent $a_{\mu \nu \alpha \beta}$ that can be chosen, but we see that this leaves generally speaking 20 independent second derivatives.

The Bianchi identities (80) can also be proven directly without going to this particular coordinate system. But the work is evidently much more cumbersome. It may be worthwhile to note that the gauge choice (79) is possible, independent of the dimension of space-time. Thus the Bianchi identities hold in arbitrary dimensions. There are other equations, to be considered later, that hold only in a space of given dimensionality.

\section{The Lagrangian for pure gravitation}

Since there is only one tensor that can be constructed from the gravitational field $h_{\mu \nu}$, namely the Riemann tensor, the problem of finding a possible Lagrangian for the gravitational field is quite straightforward. The Lagrangian can be a polynomial in the Riemann tensor; the first few possible terms can be written easily,

$$
\mathcal{L}=\sqrt{g}\left\{c_{0}+c_{1} R+c_{2} R^{2}+c_{3} R_{\mu \nu} R^{\mu \nu}+c_{4} R_{\mu \nu \alpha \beta} R^{\mu \nu \alpha \beta}+\ldots\right\} .
$$

Here $R^{\mu \nu}$ is defined by

$$
R^{\mu \nu}=g^{\mu \alpha} g^{\nu \beta} R_{\alpha \beta} .
$$

Similarly $R^{\mu \nu \alpha \beta}$. The constants $c_{0}, c_{1}$, etc. are as yet arbitrary. We must 'work out this $\&$, inserting $g_{\mu \nu}=\delta_{\mu \nu}+\kappa h_{\mu \nu}$.

Now the Riemann tensor contains second order derivatives, and the terms 
quadratic in this tensor will produce terms of the form $\partial^{2} h \partial^{2} h$. By the usual procedure this will lead to a graviton propagator behaving like $k^{-4}$, which is maybe nice for the convergence of the diagrams, but is really unacceptable because such propagators contain ghosts. In other words, allowing terms $R^{2}$ leads eventually to negative probabilities or acausal behaviour or other nonsense. Unless we are forced to for one reason or the other, we must therefore not allow such terms in the Lagrangian. We are left with two constants that can be non-zero, namely $c_{0}$ and $c_{1}$.

In terms of the fields $h_{\mu \nu}$ the Lagrangian is still very complicated. We must first find the quadratic part to see that we get the correct propagator. To this purpose we must expand $g$ and $R$ up to second order in $h$. It is of some advantage not yet to specify that $g_{\mu \nu}=\delta_{\mu \nu}$ if $h=0$. Instead we make the substitution

$$
g_{\mu \nu} \rightarrow \bar{g}_{\mu \nu}=g_{\mu \nu}+h_{\mu \nu}
$$

and develop everything to second order in $h$. Later we will set $g_{\mu \nu}=\delta_{\mu \nu}$ and replace $h$ by $k h$. The comma denotes covariant differentiation:

$$
\begin{aligned}
& \bar{g}_{\mu \nu}=g_{\mu \nu}+h_{\mu \alpha}=g_{\mu \alpha}\left(\delta_{\nu}^{\alpha}+h_{\nu}^{\alpha}\right), \\
& \bar{g}^{\mu \nu}=g^{\mu \alpha}\left(\delta_{\alpha}^{\nu}-h_{\alpha}^{\nu}+h_{\alpha}^{\beta} h_{\beta}^{\nu}\right), \\
& \sqrt{\bar{g}}=\sqrt{g}\left\{1+\frac{1}{2} h_{\alpha}{ }^{\alpha}-\frac{1}{4} h^{\alpha}{ }_{\beta} h_{\alpha}^{\beta}+\frac{1}{8}\left(h_{\alpha}^{\alpha}\right)^{2}\right\}, \\
& \bar{\Gamma}_{\mu \nu}^{\alpha}=\Gamma_{\mu \nu}^{\alpha}+\underline{\Gamma}_{\mu \nu}^{\alpha}+\underline{\underline{\Gamma}}_{\mu \nu}^{\alpha}, \\
& \underline{\Gamma}_{\mu \nu}^{\alpha}=\frac{1}{2}\left(h_{\nu, \mu}^{\alpha}+h_{\mu, \nu}^{\alpha}-h_{\mu \nu}^{, \alpha}\right), \\
& \underline{\Gamma}_{\mu \nu}^{\alpha}=-\frac{1}{2} h^{\alpha \gamma}\left(h_{\gamma \nu, \mu}+h_{\mu \gamma, \nu}-h_{\mu \nu, \gamma}\right), \\
& \underline{\Gamma}_{\mu \alpha}^{\alpha}=\frac{1}{2} h_{\alpha, \mu}^{\alpha}, \quad \underline{\Gamma}_{\mu \alpha}^{\alpha}=-\frac{1}{2} h_{\beta}^{\alpha} h_{\alpha, \mu}^{\beta}, \\
& \bar{R}_{\nu \alpha \beta}^{\mu}=R_{\nu \alpha \beta}^{\mu}+\underline{R}_{\nu \alpha \beta}^{\mu}+\underline{\underline{R}}_{\nu \alpha \beta \beta}^{\mu}, \\
& \underline{R}_{\nu \alpha \beta}^{\mu}=\frac{1}{2}\left(h_{\beta, \nu \alpha}^{\mu}-h_{\nu \beta, \alpha}^{, \mu}-h_{\alpha, \nu \beta}^{\mu}+h_{\nu \alpha, \beta}^{, \mu}\right)+\frac{1}{2} R_{\gamma \alpha \beta}^{\mu} h_{\nu}^{\gamma}+\frac{1}{2} R_{\nu \beta \alpha}^{\gamma} h_{\gamma}^{\mu} \text {, }
\end{aligned}
$$




$$
\begin{aligned}
\underline{\underline{R}}_{\nu \alpha \beta}^{\mu}= & D_{\alpha} \underline{\underline{\Gamma}}_{\nu \beta}^{\mu}-D_{\beta} \underline{\Gamma}_{\nu \alpha}^{\mu}+\underline{\Gamma}_{\beta \nu}^{\gamma} \underline{\Gamma}_{\gamma \alpha}^{\mu}-\underline{\Gamma}_{\alpha \nu}^{\gamma} \underline{\Gamma}_{\gamma \beta}^{\mu}, \\
\underline{R}_{\nu \alpha}= & \frac{1}{2}\left(h_{\beta, \nu \alpha}^{\beta}-h_{\nu, \alpha \beta}^{\beta}-h_{\alpha, \nu \beta}^{\beta}+h_{\nu \alpha, \beta}^{, \beta}\right), \\
\underline{\underline{R}}_{\nu \alpha}= & -\frac{1}{2} D_{\alpha}\left(h_{\mu}^{\beta} h_{\beta, \nu}^{\mu}\right)+\frac{1}{2} D_{\beta}\left\{h_{\gamma}^{\beta}\left(h_{\nu, \alpha}^{\gamma}+h_{\alpha, \nu}^{\gamma}-h_{\alpha \nu}^{, \gamma}\right)\right\} \\
& +\frac{1}{4}\left(h_{\beta, \nu}^{\gamma}+h_{\nu, \beta}^{\gamma}-h_{\beta \nu}^{\gamma}\right)\left(h_{\gamma, \alpha}^{\beta}+h_{\alpha, \gamma}^{\beta}-h_{\gamma \alpha}^{, \beta}\right) \\
& \quad-\frac{1}{4}\left(h_{\alpha, \nu}^{\gamma}+h_{\nu, \alpha}^{\gamma}-h_{\nu \alpha}^{, \gamma}\right) h_{\beta, \gamma}^{\beta}, \\
\underline{R}= & h_{\beta, \alpha}^{\beta, \alpha}-h_{\alpha, \beta}^{\beta, \alpha}-R_{\nu}^{\alpha} h_{\alpha}^{\nu}, \\
\underline{\underline{R}}= & -\frac{1}{2} D_{\alpha}\left(h_{\mu}^{\beta} h_{\beta}^{\mu, \alpha}\right)+\frac{1}{2} D_{\beta}\left\{h_{\nu}^{\beta}\left(2 h_{, \alpha}^{\nu \alpha}-h_{\alpha}^{\alpha, \nu}\right)\right\} \\
& +\frac{1}{4}\left(h_{\beta, \alpha}^{\nu}+h_{\alpha, \beta}^{\nu}-h_{\beta \alpha}^{, \nu}\right)\left(h_{\nu}^{\beta, \alpha}+h_{, \nu}^{\beta \alpha}-h_{\nu}^{\alpha, \beta}\right) \\
& -\frac{1}{4}\left(2 h_{, \alpha}^{\nu \alpha}-h_{\alpha}^{\alpha, \nu}\right) h_{\beta, \nu}^{\beta}-\frac{1}{2} h^{\nu \alpha} h_{\beta, \nu \alpha}^{\beta} \\
+ & \frac{1}{2} h_{\alpha}^{\nu} D_{\beta}\left(h_{\nu}^{\beta, \alpha}+h_{, \nu}^{\beta \alpha}-h_{\nu}^{\alpha, \beta}\right)+h_{\beta}^{\nu} h_{\alpha}^{\beta} R_{\nu}^{\alpha} .
\end{aligned}
$$

Omitting total derivatives this leads to the Lagrangian

$$
\begin{aligned}
\mathcal{L} & =\sqrt{g}\left[c_{0}\left\{1+\frac{1}{2} h_{\alpha}^{\alpha}-\frac{1}{4} h_{\beta}^{\alpha} h_{\alpha}^{\beta}+\frac{1}{8}\left(h_{\alpha}^{\alpha}\right)^{2}\right\}\right. \\
& +c_{1}\left\{R-\frac{1}{2} h_{\alpha}^{\alpha} R+h_{\alpha}^{\beta} R_{\beta}^{\alpha}-R\left(\frac{1}{8}\left(h_{\alpha}^{\alpha}\right)^{2}-\frac{1}{4} h_{\beta}^{\alpha} h_{\alpha}^{\beta}\right)\right. \\
& -h_{\beta}^{\nu} h_{\alpha}^{\beta} R_{\nu}^{\alpha}+\frac{1}{2} h_{\alpha}^{\alpha} h_{\beta}^{\nu} R_{\nu}^{\beta}-\frac{1}{4} h_{\alpha, \nu}^{\beta} h_{\beta}^{\alpha, \nu} \\
& \left.\left.+\frac{1}{4} h_{\alpha, \mu}^{\alpha} h_{\beta}^{\beta, \mu}-\frac{1}{2} h_{\alpha, \beta}^{\alpha} h_{\mu}^{\beta, \mu}+\frac{1}{2} h_{\beta}^{\nu, \alpha} h_{\alpha, \nu}^{\beta}\right\}\right] .
\end{aligned}
$$

If we substitute now $g_{\mu \nu}=\delta_{\mu \nu}$ we get $R=0$, and furthermore the covariant derivative becomes the ordinary derivative. Also $h_{\alpha}^{\beta}=h_{\alpha \beta}=h^{\alpha \beta}$, and $\sqrt{g}=1$. We get 


$$
\begin{aligned}
\mathcal{L}= & c_{0}\left\{1+\frac{1}{2} h_{\alpha \alpha}-\frac{1}{4} h_{\alpha \beta} h_{\alpha \beta}+\frac{1}{8} h_{\alpha \alpha} h_{\beta \beta}\right\} \\
& +c_{1}\left\{-\frac{1}{4} \partial_{\nu} h_{\alpha \beta} \partial_{\nu} h_{\alpha \beta}+\frac{1}{4} \partial_{\mu} h_{\alpha \alpha} \partial_{\mu} h_{\beta \beta}\right. \\
& \left.-\frac{1}{2} \partial_{\beta} h_{\alpha \alpha} \partial_{\mu} h_{\beta \mu}+\frac{1}{2} \partial_{\alpha} h_{\nu \beta} \partial_{\nu} h_{\alpha \beta}\right\} .
\end{aligned}
$$

Here we should still make the substitution $h \rightarrow \kappa h$.With $c_{1}=1 / \kappa^{2}$ and $c_{0}=0$ this is then precisely the Lagrangian $\mathcal{L}_{\text {sym }}$ written down before (see eq. (3), with a partial differentiation of the second term).

\section{The cosmological term}

The term $c_{0}$ is called the cosmological term. What are the experimental consequences of this term? First of all, it leads to a contribution in the classical equations ofgravity, and as such an experimental limit can be established. It is

$$
c_{0}<\left(1.23 \times 10^{-9} \mathrm{MeV}\right)^{4} .
$$

In quantum theory a non-zero $c_{0}$ implies a contribution to the propagator and the appearance of a tadpole. With the gauge breaking term $C$ of eq. (5) the Lagrangian becomes

$$
\mathcal{L}=c_{0}\left(1+\frac{1}{2} \kappa h_{\alpha \alpha}\right)+\frac{1}{2} h_{\mu \nu} V_{\mu \nu \alpha \beta}\left(\partial^{2}-c_{0} \kappa^{2}\right) h_{\alpha \beta} .
$$

This leads to almost the same propagator as before (see eq. (10)), except that $k^{2}$ is replaced by $k^{2}+c_{0} k^{2}$. This propagator therefore corresponds to the exchange of a particle with mass $c_{0} \kappa^{2}$. In first instance the gravitational force becomes a Yukawa force with a range given by this mass. However, the theory is not acceptable in this form because negative probability occurs. As is known from the treatment ofgauge theories physical sources are those for which the term

$$
h_{\mu \nu} J_{\mu \nu}
$$

is invariant under gauge transformations. To zeroth order in $K$ this means

$$
\partial_{\mu} J_{\mu \nu}=\partial_{\nu} J_{\mu \nu}=0 \text {. }
$$


Now consider two such sources, $J_{\mu \nu}$ and $J_{\alpha \beta}^{*}$ exchanging a graviton,

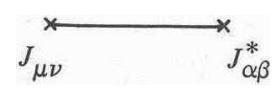

The corresponding expression is

$$
J_{\mu \nu} \frac{\delta_{\mu \alpha} \delta_{\nu \beta}+\delta_{\mu \beta} \delta_{\nu \alpha}-\delta_{\mu \nu} \delta_{\alpha \beta}}{k^{2}+m^{2}-i \epsilon} J_{\alpha \beta}^{*},
$$

with $m^{2}=c_{0} k^{2}$. At the pole $k^{2}+m^{2}=0$ the residue must be positive definite. In the $k$ restframe $\left(k=0, k_{4}=i m\right)$ we must have

$$
k_{\mu} J_{\mu \nu}=k_{\mu} J_{\nu \mu}=0
$$

or

$$
J_{4 \nu}=J_{\nu 4}=0 .
$$

Now take the following /:

$$
J_{11}=J_{22}=J_{33}=J, \quad \text { all others zero . }
$$

The residue at the pole becomes

$$
2\left(J_{11} J_{11}^{*}+J_{22} J_{22}^{*}+J_{33} J_{33}^{*}\right)-\left(J_{11}+J_{22}+J_{33}\right)^{2}=-3 J J^{*},
$$

which is unacceptable.

However, we have not treated the tadpole, and since we know that tadpoles may change masses this discussion is not complete. Usually, a tadpole is removed by a substitution of the form

$$
h_{\mu \nu} \rightarrow h_{\mu \nu}+a_{\mu \nu},
$$

and $a_{\mu \nu}$ is chosen in such a way that the terms linear in $h$ cancel in the Lagrangian. In the $a$ model, and other models with a spontaneous symmetry breakdown this may usually be achieved with constant $a$. Here this is impossible. If we want the tadpoles to cancel we may fix $a_{\mu \nu}$ from the Lagrangian given before. Now $g_{\mu \nu}=\delta_{\mu \nu}+a_{\mu \nu}$, and the condition that there are no tadpoles becomes

$$
c_{1}\left(R_{\beta}^{\alpha}-\frac{1}{2} \delta_{\beta}^{\alpha} R\right)+\frac{1}{2} c_{0} \delta_{\beta}^{\alpha}=0 .
$$


Now, if $g$ is a constant, then $R=0$, and no solution exists for non-zero $c_{0}$. Thus a space-time dependent $a_{\mu \nu}$ must be employed, and subsequently the quantum theory must be considered with everywhere this $c$-number function appearing. Up to now this has not been worked out, not even in the very simple case of a source $J_{\mu \nu}$ only, as discussed above.

\section{Scalar particles}

Here and in the following we take the cosmological constant to be zero. The next task is to try to construct gauge invariant interactions between gravitons and other fields. We will start with scalar fields.

In case that no gravitation is present the Lagrangian for a free scalar field is of the form

$$
\mathcal{L}_{\phi}=-\frac{1}{2} \partial_{\mu} \phi \partial_{\mu} \phi-\frac{1}{2} m^{2} \phi^{2} .
$$

This is not a scalar in the sense of the gravitational gauge transformations, but it is not difficult to write down an invariant Lagrangian that reduces to the above in the case of zero gravitational field. We restrict ourselves to terms quadratic in the $\phi$ fields, and will leave tadpole like terms (in $\phi$ ) out of consideration

$$
\mathscr{L}_{\phi}=\sqrt{g}\left[-\frac{1}{2} D^{\mu} \phi D_{\mu} \phi-\frac{1}{2} m^{2} \phi^{2}+a R \phi^{2}+b R_{\mu \nu} D^{\mu} \phi D^{\nu} \phi+\ldots\right] .
$$

The field $\phi$ is assigned to be a scalar under general gauge transformations. Then

$$
D_{\mu} \phi=\partial_{\mu} \phi, \quad D^{\mu} \phi=g^{\mu \nu} \partial_{\nu} \phi .
$$

As a first step we will consider only the terms linear in the gravitational field $h_{\mu \nu}$. Also the $b$ term and higher will not be considered, because they imply more than two derivatives. With the help of equations given before we find (setting $g_{\mu \nu}=\delta_{\mu \nu}$ ineqs.(83)-(96)):

$$
\begin{gathered}
\mathcal{L}_{\phi} \sim-\frac{1}{2} \partial_{\mu} \phi \partial_{\mu} \phi-\frac{1}{2} m^{2} \phi^{2}+\frac{1}{2} h_{\alpha \alpha}\left(-\frac{1}{2} \partial_{\mu} \phi \partial_{\mu} \phi-\frac{1}{2} m^{2} \phi^{2}\right) \\
+\frac{1}{2} h_{\mu \nu} \partial_{\mu} \phi \partial_{\nu} \phi+a\left(h_{\alpha \alpha} \partial^{2}-h_{\alpha \beta} \partial_{\alpha} \partial_{\beta}\right) \phi^{2} .
\end{gathered}
$$

The coupling of $h$ to the scalar field can be written in the form 
$-\kappa h_{\mu \nu} T_{\mu \nu}$,

with

$$
\begin{aligned}
T_{\mu \nu} & =\frac{1}{4} \delta_{\mu \nu}\left(\partial_{\alpha} \phi \partial_{\alpha} \phi+m^{2} \phi^{2}\right)-\frac{1}{2} \partial_{\mu} \phi \partial_{\nu} \phi \\
& -a \delta_{\mu \nu} \partial^{2} \phi^{2}+a \partial_{\mu} \partial_{\nu} \phi^{2} .
\end{aligned}
$$

We have now made the replacement $h \rightarrow \kappa h . T_{\mu \nu}$ is called the energy-momentum tensor. It contains one free parameter.

The next step is to consider the scattering of scalar particles caused by the exchange of a graviton. The Feynman rules are

$$
\begin{aligned}
& \frac{1}{(2 \pi)^{4} i} \frac{1}{k^{2}+m^{2}-i \epsilon}, \\
& k_{k}^{p} \frac{1}{(2 \pi)^{4} i} \frac{\delta_{\mu \alpha} \delta_{\nu \beta}+\delta_{\mu \beta} \delta_{\nu \alpha}-[2 /(n-2)] \delta_{\mu \nu} \delta_{\alpha \beta}}{k^{2}-i \epsilon} \\
& \left.-k_{\mu} p_{\nu}-2 a \delta_{\mu \nu} q^{2}+2 a q_{\mu} q_{\nu}\right]
\end{aligned}
$$

Consider now the scattering of one scalar particle from another. If we take two different scalar fields we have less diagrams. In fact, to order $\kappa^{2}$ we have only one diagram, see figure.

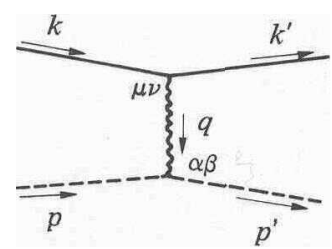

The second type of scalar is denoted by a dotted line, but couples otherwise in the same manner. Its mass will be denoted by $M$. We are mainly interested in large distance behaviour (i.e. small momentum transfer $q$ ) in the non-relati- 
vistic limit. If we neglect $k, p, k^{\prime}$ and $p^{\prime}$ compared to $m$ and $M$ the vertex simplifies greatly. We get, taking the dimension $n=4$,

$$
\begin{aligned}
& (2 \pi)^{4} i \kappa^{2}\left[-\delta_{\mu 4} \delta_{\nu 4} m^{2}\right] \frac{\delta_{\mu \alpha} \delta_{\nu \beta}+\delta_{\mu \beta} \delta_{\nu \alpha}-\delta_{\mu \nu} \delta_{\alpha \beta}}{q^{2}-i \epsilon}\left[-\delta_{\alpha 4} \delta_{\beta 4} M^{2}\right] \\
& =(2 \pi)^{4} i \kappa^{2} m^{2} M^{2} \frac{1}{q^{2}-i \epsilon} .
\end{aligned}
$$

Note that $q_{0}=k_{0}-k_{0}^{\prime}$ is of the order $q^{2}$, so that $q^{2}$ is in good approximation given by $q^{2}$. It is instructive to compare this with the scattering of two charged particles exchanging a photon. The Lagrangian is

$$
\mathcal{L}=-\left(D_{\mu} \phi\right)^{*} D_{\mu} \phi-m^{2} \phi^{*} \phi-\frac{1}{4} F_{\mu \nu} F_{\mu \nu},
$$

with now

$$
D_{\mu}=\partial_{\mu}-i e A_{\mu}, \quad F_{\mu \nu}=\partial_{\mu} A_{\nu}-\partial_{\nu} A_{\mu} .
$$

The interaction becomes

$$
i e A_{\mu}\left(\partial_{\mu} \phi^{*} \phi-\phi^{*} \partial_{\mu} \phi\right)-e^{2} A_{\mu} A_{\mu} \phi^{*} \phi .
$$

The term quadratic in the photon field is of no importance here. The relevant vertex is

$$
\int_{\mu}^{k}(2 \pi)^{4} i \cdot i e \cdot i\left(p_{\mu}-k_{\mu}\right) .
$$

The contribution of the relevant diagram is

$$
\begin{aligned}
& (2 \pi)^{4} i(i e)^{2}\left(-i k^{\prime}-i k\right)_{\mu} \frac{\delta_{\mu \nu}}{q^{2}-i \epsilon}\left(-i p^{\prime}-i p\right)_{\nu} \\
& \int_{p-\ldots p^{\prime}}^{k}=(2 \pi)^{4} i e^{2} \frac{\left(k^{\prime}+k, p^{\prime}+p\right)}{q^{2}-i \epsilon} \\
& \approx(2 \pi)^{4} i 4 e^{2} \frac{-m M}{q^{2}-i \epsilon} .
\end{aligned}
$$


This has the opposite sign compared to the gravity case: there is repulsion instead of attraction. Furthermore we see that in this approximation gravitation can be obtained from electricity by replacing $e^{2}$ by $\frac{1}{4} \kappa^{2} m M$. Notably Coulomb's law

$$
F=e^{2} / r^{2}
$$

becomes Newton's law

$$
F=\kappa^{2} m M / 4 r^{2} \text {. }
$$

From the comparison one obtains

$$
\kappa=5.9 \times 10^{-22} \mathrm{MeV}^{-1}
$$

No experiment in gravitation involves $q^{2}$ such that the constant $a$ can be measured. In fact, we see that the terms involving $a$ do not have long range (i.e. $1 / r$ ) behaviour.

From the above it may be observed that gauge invariance implies a unique coupling between gravitons and scalar particles. This is unlike in the case of electrodynamics, where the covariant derivative $D_{\mu}=\partial_{\mu}-i e A_{\mu}$ involves the charge of the particle which is still arbitrary (otherwise stated, the constant $e$ is not related to something appearing already in the photon part of the Lagrangian). In the case of gravity gauge invariance forces all couplings to be given by the same $\kappa$. This is essentially the principle ofequivalence. Given the free Lagrangian (i.e. the inertial mass of a particle) the coupling to the gravitational field is fixed.

\section{One-loop divergencies}

We will now concentrate on the calculation ofone-loop divergencies. This requires the discussion of some techniques due to 't Hooft. Important in this connection is the background field method, introduced originally by De Witt.

Suppose we have a Lagrangian describing a set ofinteracting fields $\phi_{i}$ (some of them can be the $h_{\mu \nu}$, others scalar or vector fields, etc.):

$$
\mathcal{L}=\mathcal{L}\left(\phi_{i}\right)
$$

From this Lagrangian Feynman rules can be deduced, and one loop diagrams 
can be computed. One loop diagrams contain vertices, and from each vertex precisely two lines connect via propagators to other vertices:

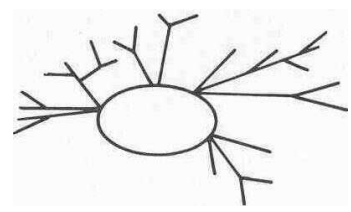

Such one loop diagrams can therefore be generated also by means of a Lagrangian containing vertices involving only two fields (and c-number functions describing the external trees). We may therefore ask to construct next to the Lagrangian (119) a Lagrangian quadratic in the fields $\phi$ such that the same one loop diagrams are generated. The answer to this question is very simple. Substitute in the Lagrangian (119) for the fields $\phi_{i}$ the sum $\widetilde{\phi}_{i}+\phi_{i}$. The $\widetilde{\phi}_{i}$ are $c$ number functions and are called the background fields. Next develop $\mathcal{L}$ up to second order in the $\phi_{i}$,

$$
\mathcal{L}\left(\widetilde{\phi}_{i}+\phi_{i}\right)=\mathcal{L}_{0}+\mathcal{L}_{1}+\mathcal{L}_{2}+\ldots
$$

The Lagrangian $\mathcal{L}_{2}$, given by

$$
\mathcal{L}_{2}=\left.\frac{1}{2} \frac{\delta^{2} \mathcal{L}}{\delta \phi_{i} \delta \phi_{j}}\right|_{\phi=\widetilde{\phi}} \phi_{i} \phi_{j},
$$

is precisely the Lagrangian asked for. We leave it to the reader to check this fact, for instance by considering a number of simple examples. We emphasize that no reference is made as to whether the fields $\widetilde{\phi}$ obey the classical equations of motion. Since tree diagrams with the outer legs on mass shell are described by fields obeying the classical equations of motion the use of $\widetilde{\phi}$ that are such solutions is like going on mass shell with the external legs.

Let us now consider some Lagrangians. First

$$
\mathcal{L}_{2}(\phi)=-\frac{1}{2} \partial_{\mu} \phi_{i} \partial_{\mu} \phi_{i}+\phi_{i} N_{i j}^{\mu} \partial_{\mu} \phi_{j}+\frac{1}{2} \phi_{i}\left(M_{i j}-\partial_{\mu} N_{i j}^{\mu}\right) \phi_{j} .
$$

In here $N$ and $M-\partial_{\mu} N^{\mu}$ are antisymmetric and symmetric in the indices $i$ and j. $M$ and $N$ are classical functions of space time. Matters are simplified by means of the so-called doubling trick. We add to this Lagrangian the identical one but with other fields $\bar{\phi}_{i}$. This clearly amounts to doubling the results since the number of diagrams doubles. Writing now 


$$
\phi_{i}=\sqrt{\frac{1}{2}}\left(A_{i}+A_{i}^{*}\right), \quad \phi_{i}=-i \sqrt{\frac{1}{2}}\left(A_{i}-A_{i}^{*}\right),
$$

we obtain

$$
\mathcal{L}_{2}(\phi)+\mathcal{L}_{2}(\bar{\phi})=-\partial_{\mu} A_{i}^{*} \partial_{\mu} A_{i}+2 A_{i}^{*} N_{i j}^{\mu} \partial_{\mu} A_{i}+A_{i}^{*} M_{i j} A_{j} .
$$

Instead of (120) we will thus consider the Lagrangian

$$
\mathcal{L}(\phi)=-\partial_{\mu} \phi_{i}^{*} \partial_{\mu} \phi_{i}+2 \phi_{i}^{*} N_{i j}^{\mu} \partial_{\mu} \phi_{j}+\phi_{i}^{*} M_{i j} \phi_{j},
$$

and to get the results in the case of the Lagrangian (120) we only need to divide by two.

To limit the work as much as possible we rewrite this Lagrangian now as follows:

$$
\mathcal{L}(\phi)=-D_{\mu} \phi^{*} D_{\mu} \phi+\phi^{*} Q \phi,
$$

with

$$
\left(D_{\mu} \phi\right)_{i}=\partial_{\mu} \phi_{i}+P_{\mu}^{i j} \phi_{j} .
$$

This coincides with (121) if

$$
\begin{aligned}
& P_{\mu}^{i j}=N_{\mu}^{i j}, \\
& Q_{i j}+\partial_{\mu} P_{\mu}^{i j}+P_{\mu}^{i m} P_{\mu}^{m j}=M_{i j} .
\end{aligned}
$$

Note that $N$ and also $P$ are taken to be antisymmetric in the indices $i, j$.

Formally, the Lagrangian (122) possesses a symmetry. It is invariant for the infinitesimal transformation

$$
\begin{aligned}
& \phi \rightarrow \phi-\Lambda \phi, \quad \Lambda^{i j}=-\Lambda^{i i}, \\
& Q \rightarrow Q+[Q, \Lambda], \\
& P_{\mu} \rightarrow P_{\mu}+\left[P_{\mu}, \Lambda\right]+\partial_{\mu} \Lambda .
\end{aligned}
$$

For instance, 


$$
\begin{aligned}
& D_{\mu} \phi \rightarrow D_{\mu} \phi-\Lambda \partial_{\mu} \phi-\left(\partial_{\mu} \Lambda\right) \phi-P_{\mu} \Lambda \phi+\left[P_{\mu}, \Lambda\right] \phi+\left(\partial_{\mu} \Lambda\right) \phi \\
& \quad=D_{\mu} \phi-\Lambda \partial_{\mu} \phi-\Lambda P_{\mu} \phi \\
& \quad=(1-\Lambda) D_{\mu} \phi, \\
& D_{\mu} \phi^{*} \rightarrow(1-\Lambda) D_{\mu} \phi^{*}=D_{\mu} \phi^{*}(1+\Lambda) .
\end{aligned}
$$

Obviously $D_{\mu} \phi^{*} D_{\mu} \phi$ is invariant up to terms of order $\Lambda^{2}$.

Since the Lagrangian is invariant also the counterterms (the pole terms in the dimensional regularization method) are invariant. Since these counterterms will be made up from the $P$ and the $Q$ it is therefore of help if we know what invariants can be made.

First, if $Q_{1}, Q_{2}, \ldots$ are quantities that transform like $Q$ then also the product ofthese quantities transforms like $Q$. The proofis simple; the finite form of the transformation of $Q$ is evidently

$$
Q \rightarrow \mathrm{e}^{-\Lambda} Q \mathrm{e}^{\Lambda},
$$

and the statement is obviously true. Furthermore, the trace of a quantity transforming like $Q$ is invariant because

$$
\operatorname{Tr}\left(\mathrm{e}^{-\Lambda} Q \mathrm{e}^{\Lambda}\right)=\operatorname{Tr}\left(Q \mathrm{e}^{\Lambda} \mathrm{e}^{-\Lambda}\right)=\operatorname{Tr}(Q) .
$$

The finite form of the transformation of the $P_{\mu}$ is

$$
P_{\mu} \rightarrow \mathrm{e}^{-\Lambda} P_{\mu} \mathrm{e}^{\Lambda}+\left(\partial_{\mu} \mathrm{e}^{-\Lambda}\right) \mathrm{e}^{\Lambda} .
$$

Indeed, if we substitute in this equation for $P$ the same formula but with a $\bar{\Lambda}$, we get

$$
P_{\mu} \rightarrow \mathrm{e}^{-\Lambda} \mathrm{e}^{-\bar{\Lambda}} P_{\mu} \mathrm{e}^{\bar{\Lambda}} \mathrm{e}^{\Lambda}+\mathrm{e}^{-\Lambda}\left(\partial_{\mu} \mathrm{e}^{-\bar{\Lambda}}\right) \mathrm{e}^{\bar{\Lambda}} \mathrm{e}^{\Lambda}+\left(\partial_{\mu} \mathrm{e}^{-\Lambda}\right) \mathrm{e}^{\Lambda},
$$

with

$$
\partial_{\mu} e^{-\Lambda} e^{\Lambda}=\partial_{\mu} e^{-\Lambda} e^{-\bar{\Lambda}} e^{\bar{\Lambda}} e^{\Lambda},
$$

and we see that this is again of the same form but with

$$
\mathrm{e}^{\bar{\Lambda}} \mathrm{e}^{\Lambda} \quad \text { instead of } \quad \mathrm{e}^{\Lambda} .
$$


Actually, all this is very much like in a Yang-Mills theory. In SU(2) for example the finite form for the transformation of a Yang-Mills vector field is

$$
W_{\mu}^{a} \rightarrow f_{a b} W_{\mu}^{b}-\frac{1}{2 g} \epsilon_{a b c}\left(\partial_{\mu} f\right)_{c d}\left(f^{-1}\right)_{d b},
$$

where the $3 \times 3$ matrix $f_{a b}$ is a rotation in isospin space depending on space time. If we introduce the $2 \times 2$ matrices

$$
W_{\mu}=\sum W_{\mu}^{a} \tau^{a},
$$

then we get very similar equations.

Turning back to our $P$ and $Q$ we now easily demonstrate that $\varrho_{\mu \nu}$ defined by

$$
g_{\mu \nu}=\partial_{\mu} P_{\nu}-\partial_{\nu} P_{\mu}+\left[P_{\mu}, P_{\nu}\right]
$$

transforms like $Q$,

$$
g_{\mu \nu} \rightarrow g_{\mu \nu}+\left[g_{\mu \nu}, \Lambda\right] .
$$

Furthermore, for any quantity transforming like $Q$ we may define the covariant derivative,

$$
\bar{D}_{\mu} X \equiv \partial_{\mu} X+\left[P_{\mu}, X\right] \text {. }
$$

Then $\bar{D}_{\mu} X$ transforms like $Q$,

$$
\bar{D}_{\mu} X \rightarrow \bar{D}_{\mu} X+\left[\bar{D}_{\mu} X, \Lambda\right] .
$$

It is now an easy matter to write invariants

$$
\operatorname{Tr}(Q), \quad \operatorname{Tr}\left(Q^{2}\right), \quad \operatorname{Tr}\left(\bar{D}_{\mu} \bar{D}_{\mu} Q\right), \quad \operatorname{Tr}\left(\varrho_{\mu \nu} \Theta_{\mu \nu}\right), \quad \text { etc. }
$$

Returning now to the Lagrangian (121) we have the following Feynman rules

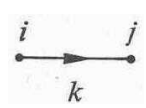

$$
\frac{\delta_{i j}}{k^{2}-i \epsilon} \frac{1}{(2 \pi)^{4} i},
$$

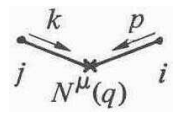

$$
(2 \pi)^{4} i 2 N_{i j}^{\mu}(q) k_{\mu}, \quad q+k+p=0,
$$

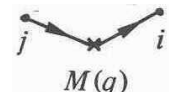

$$
(2 \pi)^{4} i M_{i j}(q) \text {. }
$$


The following one-loop diagrams are divergent:
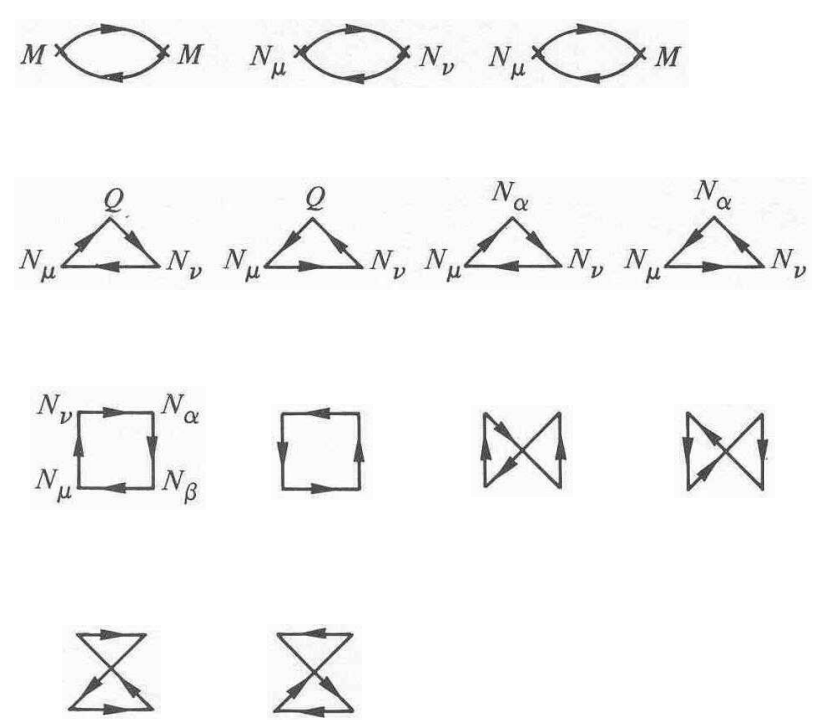

We see that the counter Lagrangian is at most quadratic in $M$ and quartic in $N$. In terms of $Q$ and $P$ it must therefore be of the form

$$
\mathcal{L}_{\mathrm{c}}=\alpha \operatorname{Tr}\left(Q^{2}\right)+\beta \operatorname{Tr}\left(g_{\mu \nu} g_{\mu \nu}\right) .
$$

Note that

$$
\left(\bar{D}_{\mu} \bar{D}_{\nu}-\bar{D}_{\nu} \bar{D}_{\mu}\right) X=\left[\Theta_{\mu \nu}, X\right],
$$

so that we need not consider $\bar{D}_{\mu} \bar{D}_{\nu} \rho_{\mu \nu}$.

By computing the first and second diagrams it is easy to determine the coefficients $\alpha$ and $\beta$. The first diagram leads to the expression

$$
\int \mathrm{d}_{n} p \frac{M_{i j}(k) M_{j i}(-k)}{p^{2}(p+k)^{2}} .
$$

The divergent part is

$$
M_{i j} M_{j i} \int \mathrm{d}_{n} p \frac{1}{p^{4}} \equiv Z_{0} \operatorname{Tr}\left(M^{2}\right),
$$


with

$$
Z_{0}=-\frac{2 i \pi^{2}}{n-4}
$$

The corresponding counterterm is

$$
\begin{aligned}
& \frac{-Z_{0}}{(2 \pi)^{4} i} \frac{1}{2} \operatorname{Tr}\left(M^{2}\right) \equiv \frac{1}{\epsilon} \operatorname{Tr}\left(\frac{1}{2} M^{2}\right), \\
& \epsilon=8 \pi^{2}(n-4) .
\end{aligned}
$$

The factor $\frac{1}{2}$ is because a term $\lambda \phi^{2} X$ in the Lagrangian gives the vertex

$$
\text { - = }=\text { X } X \quad(2 \pi)^{4} i 2 \lambda \text {. }
$$

Thus the coefficient $\alpha$ is now determined to be $1 / 2 \epsilon$. The coefficient $\beta$ can be determined from the two $N$ diagram

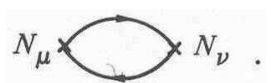

The corresponding expression is

$$
4 \operatorname{Tr}\left(N_{\mu} N_{\nu}\right) \int \mathrm{d}_{n} p \frac{-p_{\mu}(p+k)_{\nu}}{p^{2}(p+k)^{2}} .
$$

Developing the denominator and retaining only logarithmic divergencies

$$
\begin{aligned}
& \int \mathrm{d}_{n} p \frac{-p_{\mu}(p+k)_{\nu}}{p^{2}(p+k)^{2}}=-\int \mathrm{d}_{n} p \frac{p_{\mu} p_{\nu}+p_{\mu} k_{\nu}}{p^{4}}\left\{1-\frac{2 p k+k^{2}}{p^{2}}+\frac{4(p k)^{2}}{p^{4}}\right\} \\
& =-Z_{0}\left\{-\frac{1}{4} k^{2} \delta_{\mu \nu}-\frac{1}{2} k_{\mu} k_{\nu}+k_{\alpha} k_{\beta} \frac{4}{24}\left(\delta_{\alpha \beta} \delta_{\mu \nu}+\delta_{\alpha \mu} \delta_{\beta \nu}+\delta_{\alpha \nu} \delta_{\beta \mu}\right)\right\} \\
& =Z_{0}\left\{\frac{1}{12} k^{2} \delta_{\mu \nu}+\frac{1}{6} k_{\mu} k_{\nu}\right\} .
\end{aligned}
$$

The corresponding term in $\mathscr{L}_{\mathrm{c}}$ is

$$
\mathcal{L}=\frac{1}{\epsilon} \operatorname{Tr}\left\{\frac{1}{6} \partial_{\alpha} N_{\mu} \partial_{\alpha} N_{\mu}+\frac{1}{3} \partial_{\mu} N_{\mu} \partial_{\nu} N_{\nu}\right\} .
$$


Eqs. (143) and (148) are sufficient to determine the coefficients $\alpha$ and $\beta . W e$ get for the counter Lagrangian

$$
\begin{aligned}
& \mathscr{L}_{\mathrm{c}}=\frac{1}{\epsilon} \operatorname{Tr}\left(\frac{1}{2} Q^{2}+\frac{1}{12} \mathcal{G}_{\mu \nu} \mathcal{G}_{\mu \nu}\right), \\
& Q=M-N_{\mu} N_{\mu}-\partial_{\mu} N_{\mu}, \\
& \mathcal{g}_{\mu \nu}=\partial_{\mu} N_{\nu}-\partial_{\nu} N_{\mu}+N_{\mu} N_{\nu}-N_{\nu} N_{\mu} .
\end{aligned}
$$

We need the counter Lagrangian for an even more general case, namely,

$$
\mathcal{L}=\sqrt{g}\left(-\partial_{\mu} \phi^{*} g^{\mu \nu} \partial_{\nu} \phi+2 \phi^{*} N^{\mu} \partial_{\mu} \phi+\phi^{*} M \phi\right) .
$$

This is considerably more complicated. Again we define

$$
D_{\mu} \phi^{i}=\partial_{\mu} \phi^{i}+P_{\mu}^{i j} \phi^{j} .
$$

We will no more write the indices $i, j$. Furthermore,

$$
\begin{aligned}
& D^{\mu} \phi^{*}=g^{\mu \nu} D_{\nu} \phi, \\
& D^{\mu} \phi D_{\mu} \phi=g^{\mu \nu}\left(\partial_{\mu} \phi^{*} \partial_{\nu} \phi\right)+g^{\mu \nu}\left(\partial_{\mu} \phi^{*} P_{\nu} \phi\right)+g^{\mu \nu}\left(P_{\mu} \phi^{*} \partial_{\nu} \phi\right) .
\end{aligned}
$$

Now remember that $\partial_{\mu} \phi$ is a covariant derivative in the sense of gravitational gauge invariance. We may therefore partially integrate forgetting the factor $\sqrt{g}$, but treating $P_{\mu}$ as a covariant four-vector. Moreover, $D_{\alpha} g_{\mu \nu}=0$. Thus we get

$$
\sqrt{g}\left(D^{\mu} \phi D_{\mu} \phi\right) \rightarrow g^{\mu \nu}\left(\partial_{\mu} \phi^{*} \partial_{\nu} \phi\right)-2 g^{\mu \nu} \phi^{*} P_{\nu} \partial_{\mu} \phi-\phi^{*}\left(D^{\mu} P_{\mu}\right) \phi .
$$

Thus all invariance considerations hold as before. There is one difference: there are now more invariants to be considered, namely the Riemann scalars, etc. Now we can have things like

$$
\operatorname{Tr}(Q R), \quad R^{2} \operatorname{Tr}(\mathbb{I}), \quad R^{\mu \nu} R_{\mu \nu} \operatorname{Tr}(\mathbb{I}) .
$$

Moreover, $\mathcal{L}_{\mathrm{c}}$ will now contain a factor $\sqrt{g}$. The answer is 


$$
\mathcal{L}_{\mathrm{c}}=\frac{\sqrt{g}}{\epsilon} \operatorname{Tr}\left[\frac{1}{2}\left(Q-\frac{1}{6} R\right)^{2}+\frac{1}{12} g^{\mu \nu} g_{\mu \nu}+\frac{1}{60} \mathbb{I}\left(R_{\mu \nu} R^{\mu \nu}-\frac{1}{3} R^{2}\right)\right] .
$$

To prove this equation we must show the factors $\frac{1}{6} Q R, \frac{1}{60} R_{\mu \nu} R^{\mu \nu}$ and $\frac{1}{180} R^{2}$. No term of the form

$$
R_{\mu \nu \alpha \beta} R^{\mu \nu \alpha \beta}
$$

needs to be considered, because in four-dimensional space it can be reduced

$$
\begin{array}{r}
R_{\mu \nu \alpha \beta \beta} R^{\mu \nu \alpha \beta}-4 R_{\mu \nu} R^{\mu \nu}+R^{2}=\text { total derivative } \\
\quad \text { (in four dimensions). }
\end{array}
$$

This equation will be proven later.

The coefficient of the term $Q R$ can be determined readily by choosing a particular $g$. We take

$$
g_{\mu \nu}=\delta_{\mu \nu} F, \quad \sqrt{g}=F^{2}, \quad F=1-f,
$$

where/is an arbitrary function ofspace-time. The Lagrangian (150) may be rewritten,

$$
\mathcal{L}=\phi^{*} F \partial^{2} \phi+2 \phi^{*}\left(F^{2} N^{\mu}+\frac{1}{2} \partial_{\mu} F\right) \partial_{\mu} \phi+\phi^{*} F^{2} M \phi .
$$

Now substitute $\phi^{*} \rightarrow \phi^{*} F^{-1}$. This does not change anything in the diagrams. Weget

$$
\mathcal{L}=\phi^{*} \partial^{2} \phi+2 \phi^{*}\left(F N^{\mu}+\frac{1}{2} F^{-1} \partial_{\mu} F\right) \partial_{\mu} \phi+\phi^{*} F M \phi .
$$

This $\mathcal{L}$ is precisely of the form studied with the substitution

$$
\begin{aligned}
& M \rightarrow F M, \\
& N^{\mu} \rightarrow F N^{\mu}+\frac{1}{2} F^{-1} \partial_{\mu} F=N_{\mu}+\frac{1}{2} F^{-1} \partial_{\mu} F .
\end{aligned}
$$

We must now compute $R$ and $R_{\mu \nu}$ for this particular $g$. With

$$
f_{\alpha}=\partial_{\alpha} f, \quad f_{\alpha \beta}=\partial_{\alpha} \partial_{\beta} f,
$$

we find 


$$
\begin{aligned}
\Gamma_{\mu \nu}^{\alpha}= & -\frac{1}{2} F^{-1}\left(\delta_{\mu}^{\alpha} f_{\nu}+\delta_{\nu}^{\alpha} f_{\mu}-\delta_{\mu \nu} f^{\alpha}\right), \\
D_{\mu} Z^{\alpha}= & \partial_{\mu} Z^{\alpha}+\Gamma_{\mu \nu}^{\alpha} Z^{\nu} \\
& =\partial_{\mu} Z^{\alpha}-\frac{1}{2} F^{-1}\left(\delta_{\mu}^{\alpha} f_{\nu} Z^{\nu}+f_{\mu} Z^{\alpha}-f^{\alpha} Z_{\mu}\right), \\
R_{\nu \alpha \beta}^{\mu}= & -\frac{1}{2} F^{-1}\left(\delta_{\beta}^{\mu} f_{\alpha \nu}-\delta_{\alpha}^{\mu} f_{\beta \nu}-\delta_{\nu \beta} f_{\alpha}^{\mu}+\delta_{\nu \alpha} f_{\beta}^{\mu}\right) \\
& -\frac{1}{4} F^{-2}\left(3 \delta_{\beta}^{\mu} f_{\alpha} f_{\nu}-3 \delta_{\alpha}^{\mu} f_{\beta} f_{\nu}-3 \delta_{\nu \beta} f_{\alpha} f^{\mu}\right. \\
& \left.+3 \delta_{\nu \alpha} f_{\beta} f^{\mu}+\delta_{\beta \nu} \delta_{\alpha}^{\mu} f^{\gamma} f_{\gamma}-\delta_{\alpha \nu} \delta_{\beta}^{\mu} f^{\gamma} f_{\gamma}\right) .
\end{aligned}
$$

By contraction

$$
\begin{aligned}
& R_{\nu \alpha}=-\frac{1}{2} F^{-1}\left(2 f_{\alpha \nu}+\delta_{\alpha \nu} f_{\gamma \gamma}\right)-\frac{3}{2} F^{-2} f_{\alpha} f_{\nu}, \\
& R=-3 F^{-2} f_{\gamma \gamma}-\frac{3}{2} F^{-3} f_{\gamma} f_{\gamma}, \\
& R_{\nu \alpha} R^{\nu \alpha}=F^{-4}\left(f_{\alpha \nu} f_{\alpha \nu}+2 f_{\alpha \alpha} f_{\nu \nu}\right) \\
& \quad+F^{-5}\left(3 f_{\alpha \nu} f_{\alpha} f_{\nu}+\frac{3}{2} f_{\alpha \alpha} f_{\nu} f_{\nu}\right)+\frac{9}{4} F^{-6} f_{\alpha} f_{\alpha} f_{\nu} f_{\nu}, \\
& R^{2}=9 F^{-4} f_{\alpha \alpha} f_{\nu \nu}+9 F^{-5} f_{\nu \nu} f_{\alpha} f_{\alpha}+\frac{9}{4} F^{-6} f_{\alpha} f_{\alpha} f_{\nu} f_{\nu} .
\end{aligned}
$$

Now for this $g$ one verifies easily eq. (156). However, for this $g$ also

$$
\sqrt{g}\left(R_{\mu \nu} R^{\mu \nu}-\frac{1}{3} R^{2}\right)=\text { total derivative . }
$$

We can therefore determine the counter Lagrangian $\mathcal{L}$ only up to terms of this kind.

Comparing the result eq. (149) with the substitutions (160) to the Lagrangian (155) with the appropriate expressions (166) and (168) for $R$ and $R^{2}$ shows that indeed in the combination $Q+\alpha R$ the coefficient $\alpha$ must be taken as $\frac{1}{6}$. Moreover the term $R^{2}$ has the factor $\frac{1}{72}$, and we have determined Lup to an expression of the form(169) (in principle one could have additional factors $R^{2}$ ). 
To determine the last unknown coefficient there is nothing to do but to compute another diagram for some special case. We consider a Lagrangian without $M$ and $N$,

$$
\mathcal{L}=\sqrt{g}\left(-\partial_{\mu} \phi^{*} g^{\mu \nu} \partial_{\nu} \phi\right)
$$

With $g_{\mu \nu}=\delta_{\mu \nu}+h_{\mu \nu}$ we must expand up to first order in $h$,

$$
\mathcal{L}=-\partial_{\mu} \phi^{*} \partial_{\mu} \phi+\partial_{\mu} \phi^{*}\left(h^{\mu \nu}-\frac{1}{2} \delta^{\mu \nu} h^{\alpha \alpha}\right) \partial_{\nu} \phi .
$$

With

$$
s_{\mu \nu}=h^{\mu \nu}-\frac{1}{2} \delta^{\mu \nu} h^{\alpha \alpha}
$$

it will be sufficient to compute a graph with two $s$ vertices. Terms of order $h^{2}$ in the Lagrangian do not contribute for the following reason. We are working to order $h^{2}$ in the counter Lagrangian, since no more is needed to determine the last coefficient. Now a term $h^{2} \phi^{2}$ in the Lagrangian is already of order $h^{2}$ and the diagrams of order $h^{2}$ involving this vertex cannot involve any other vertex and are therefore of the tadpole type

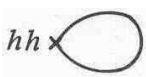

Such diagrams do not contain logarithmic divergencies, and may be taken to be zero.

We must finally compute the divergence of the diagram with two s vertices

Vertex:
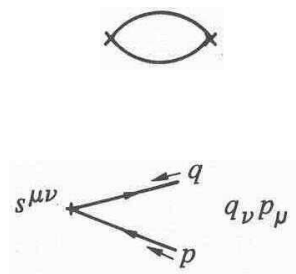

The diagram

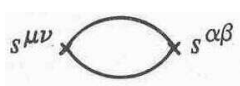

gives rise to the integral

$$
s^{\mu \nu}{ }_{s}^{\alpha \beta} \int \mathrm{d}_{n} p \frac{p_{\mu}(p+k)_{\nu} p_{\alpha}(p+k)_{\beta}}{p^{2}(p+k)^{2}} .
$$


The resulting counter Lagrangian is

$$
\begin{aligned}
\mathcal{L}_{\mathrm{c}}= & \frac{1}{2}\left[\frac{1}{240} \partial^{2} s_{\mu \mu} \partial^{2} s_{\alpha \alpha}+\frac{1}{120} \partial^{2} s_{\mu \alpha} \partial^{2} s_{\mu \alpha}+\frac{1}{30} \partial^{2} s_{\mu \mu} \partial_{\alpha} \partial_{\beta} s_{\alpha \beta}\right. \\
& \left.-\frac{1}{60} \partial_{\alpha} \partial_{\nu} s_{\mu \nu} \partial_{\alpha} \partial_{\beta} s_{\mu \beta}+\frac{1}{30} \partial_{\mu} \partial_{\nu} s_{\mu \nu} \partial_{\alpha} \partial_{\beta} s_{\alpha \beta}\right] .
\end{aligned}
$$

Next we must substitute eq. (172) and compare with terms $R^{2}, R_{\mu \nu} R^{\mu \nu}$ where we must evaluate first these terms to second order in $h$,

$$
\begin{aligned}
& R_{\nu \alpha}=\frac{1}{2}\left(\partial_{\alpha} \partial_{\nu} h_{\mu \mu}-\partial_{\nu} \partial_{\mu} h_{\alpha \mu}-\partial_{\alpha} \partial_{\mu} h_{\nu \mu}+\partial^{2} h_{\nu \alpha}\right), \\
& R_{\alpha \beta} R^{\alpha \beta}=\frac{1}{4}\left(\partial^{2} h_{\mu \mu} \partial^{2} h_{\alpha \alpha}+\partial^{2} h_{\mu \alpha} \partial^{2} h_{\mu \alpha}-2 \partial^{2} h_{\mu \mu} \partial_{\alpha} \partial_{\beta} h_{\alpha \beta}\right. \\
& \left.\quad-2 \partial_{\alpha} \partial_{\nu} h_{\mu \nu} \partial_{\alpha} \partial_{\beta} h_{\mu \beta}+2 \partial_{\mu} \partial_{\nu} h_{\mu \nu} \partial_{\alpha} \partial_{\beta} h_{\alpha \beta}\right), \\
& R=\partial^{2} h_{\mu \mu}-\partial_{\alpha} \partial_{\mu} h_{\alpha \mu}, \\
& R^{2}=\partial^{2} h_{\mu \mu} \partial^{2} h_{\alpha \alpha}-2 \partial^{2} h_{\mu \mu} \partial_{\alpha} \partial_{\beta} h_{\alpha \beta}+\partial_{\alpha} \partial_{\beta} h_{\alpha \beta} \partial_{\mu} \partial_{\nu} h_{\mu \nu}
\end{aligned}
$$

The result is

$$
\mathcal{L}_{\mathrm{c}}=\frac{\sqrt{g}}{\epsilon}\left[\frac{1}{72} R^{2}+\frac{1}{60}\left(R_{\mu \nu} R^{\mu \nu}-\frac{1}{3} R^{2}\right)\right],
$$

which shows the correctness of the general counter Lagrangian(155).

\section{Divergencies with external gravitational field}

The results of the preceding section may be summarized as follows. To the Lagrangian

$$
\mathcal{L}=\sqrt{g}\left(-\partial_{\mu} \phi^{*} g^{\mu \nu} \partial_{\nu} \phi+2 \phi^{*} N^{\mu} \partial_{\mu} \phi+\phi^{*} M \phi\right)
$$

(where $g^{\mu \nu}, N^{\mu}$ and $M$ are c-number functions of space-time) corresponds the one-loop counter Lagrangian

$$
\mathcal{L}_{\mathrm{c}}=\frac{1}{2} \sqrt{g} \operatorname{Tr}\left\{\frac{1}{2}\left(Q-\frac{1}{6} R\right)^{2}+\frac{1}{12} \varrho^{\mu \nu} \varrho_{\mu \nu}+\frac{1}{60}\left(R_{\mu \nu} R^{\mu \nu}-\frac{1}{3} R^{2}\right)\right\},
$$


with

$$
\begin{aligned}
& Q=M-N_{\mu} N^{\mu}-D_{\mu} N^{\mu}, \\
& g_{\mu \nu}=D_{\mu} N_{\nu}-D_{\nu} N_{\mu}+\left[N_{\mu}, N_{\nu}\right] .
\end{aligned}
$$

Here $D_{\mu}$ is the covariant derivative involving $g_{\mu \nu}$. Also $R$ and $R_{\mu \nu}$ are constructed from $g_{\mu \nu}$. From this formula we can already draw some important conclusions. Suppose that we consider a theory of scalar particles interacting with themselves and with an external gravitational field. This external field is represented by $g_{\mu \nu}=\delta_{\mu \nu}+\kappa h_{\mu \nu}$. To zeroth order in the gravitational coupling constantthedivergenciesaregivenby $\mathcal{L}_{\mathrm{c}}$ ofeq.(181), with $R=R_{\mu \nu}=0$ and $\sqrt{ } g=1$. To first order in $K$ we have, from $\sqrt{ } g$, essentially the same divergencies and there is a new divergence from the term $Q R$, where $R$ is worked out to first order in $K$.

The very nice thing is now that this new divergence can be cancelled. If, in eq. (180) $M$ is replaced by $M+\frac{1}{6} R$ then in eq. (181) the term

$$
\left(Q-\frac{1}{6} R\right)^{2} \rightarrow Q^{2}
$$

This suggests that the free constant $a$ in sect. 13, eq. (112) should be taken as $\frac{1}{12}$. This prevents the occurrence of new divergencies up to order $\kappa$. The choice of $a=\frac{1}{12}$ leads to an energy-momentum tensor that is often called the improved energy-momentum tensor, and was in this context first proposed by Callan, Coleman and Jackiw, A. of Phys. 59 (1972) 42.

However, to order $\kappa^{2}$ we have still the combination $R_{\mu \nu} R^{\mu \nu}-\frac{1}{3} R^{2}$. This represents divergencies in the theory ofgravitation caused by closed loops of scalars. Now to order $\kappa^{2}$ we also may get contributions from vector particles, fermions and the gravitons themselves, so the consideration of these terms here is not very meaningful.

As a first step in a more complete treatment we may now consider quantized scalar and gravitational fields together. The case ofpure gravitation is then easily deduced as well.

16. Divergencies with quantized gravitational field

We will consider the simplified case

$$
\overline{\mathcal{L}}=\sqrt{\bar{g}}\left(-\bar{R}-\frac{1}{2} \partial_{\mu} \bar{\phi} \bar{g}^{\mu \nu} \partial_{\nu} \bar{\phi}\right) .
$$


The bar on the various quantities is for notational reasons. We have not employed the improved energy-momentum tensor, but that can be done rather easily later. Furthermore we suppose units to be such that $\kappa=1$. Effectively this means that our unit of energy is given by $1 / \kappa=1.724 \times 10^{21} \mathrm{MeV}$, a rather large value. It shows in fact the irrelevance of gravitation for elementary particle physics insofar as no matters of principle are involved.

Before we get to computing the equivalent second order Lagrangian we must discuss the question of gauge invariance. The first step is that we write everywhere

$$
\bar{g}_{\mu \nu}=g_{\mu \nu}+h_{\mu \nu}, \quad \quad \phi=\widetilde{\phi}+\phi,
$$

where $g_{\mu \nu}$ and $\widetilde{\phi}$ are background fields.

The Lagrangian(183) with the substitution(184) is invariant for the general gauge transformation

$$
\bar{g}_{\mu \nu} \rightarrow \bar{g}_{\mu \nu}+\bar{g}_{\alpha \nu} \partial_{\mu} \eta^{\alpha}+\bar{g}_{\mu \alpha} \partial_{\nu} \eta^{\alpha}+\eta^{\alpha} \partial_{\alpha} \bar{g}_{\mu \nu}
$$

The replacement $\partial \rightarrow D$ where $D$ is constructed from $g$, not $\bar{g}$, may be done: all $\Gamma$ terms cancel. We so get, using eq. (184),

$$
h_{\mu \nu} \rightarrow h_{\mu \nu}+\left(g_{\alpha \nu}+h_{\alpha \nu}\right) D_{\mu} \eta^{\alpha}+\left(g_{\mu \alpha}+h_{\mu \alpha}\right) D_{\nu} \eta^{\alpha}+\eta^{\alpha} D_{\alpha} h_{\mu \nu} .
$$

Similarly,

$$
\phi \rightarrow \phi+\eta^{\alpha} D_{\alpha}(\tilde{\phi}+\phi)
$$

Again, $D$ involves $g$, not $\bar{g}=g+h$. The importance of introducing covariance with respect to the background field $g$ will become clear: the counter Lagrangian shall be invariant with respect to general gauge transformations of the background fields $g$ and $\widetilde{\phi}$, simply because the second order $\mathcal{L}$, by a properly chosen gauge breaking term (to break the gauge invariances with respect to the quantum fields eqs. (186), (187)) will possess that invariance. This is the beauty of the background field method: it offers the possibility of directly profiting from the symmetry of the theory.

The first and second order variation of the various quantities has been given before. Simplifying things as much as possible using partial integration (omitting total derivatives) one finally obtains on developing $\mathcal{L}$ from eq. (183),

$$
\overline{\mathcal{L}}=\mathcal{L}+\underline{\mathcal{L}}+\underline{\underline{\mathcal{L}}}+\mathrm{O}\left(\phi^{3}, h^{3}, \phi^{2} h, \phi h^{2}\right),
$$




$$
\begin{aligned}
\underline{\mathcal{L}} & =\sqrt{g}\left(-\frac{1}{2} h_{\alpha}^{\alpha} R-\frac{1}{4} \partial_{\mu} \widetilde{\phi} g^{\mu \nu} \partial_{\nu} \widetilde{\phi} h_{\alpha}^{\alpha}+h_{\alpha}^{\beta} R_{\beta}^{\alpha}\right. \\
& \left.+\frac{1}{2} h^{\mu \nu} \partial_{\mu} \widetilde{\phi} \partial_{\nu} \widetilde{\phi}-\partial_{\mu} \phi g^{\mu \nu} \partial_{\nu} \widetilde{\phi}\right), \\
\underline{\underline{\mathcal{L}}} & =\sqrt{g}\left[-\frac{1}{2} \partial_{\mu} \phi \partial_{\nu} \widetilde{\phi}\left(g^{\mu \nu} h_{\alpha}^{\alpha}-2 h^{\mu \nu}\right)-\frac{1}{2} \partial_{\mu} \widetilde{\phi} \partial_{\nu} \widetilde{\phi}\right. \\
& \times\left(h_{\alpha}^{\mu} h^{\alpha \nu}-\frac{1}{2} h_{\alpha}^{\alpha} h^{\mu \nu}\right)-\frac{1}{2} \partial_{\mu} \phi g^{\mu \nu} \partial_{\nu} \phi-\left(\frac{1}{8}\left(h_{\alpha}^{\alpha}\right)^{2}-\frac{1}{4} h_{\beta}^{\alpha} h_{\alpha}^{\beta}\right) \\
& \times\left(R+\frac{1}{2} \partial_{\mu} \widetilde{\phi} g^{\mu \nu} \partial_{\nu} \widetilde{\phi}\right)-h_{\beta}^{\nu} h_{\alpha}^{\beta} R_{\nu}^{\alpha}+\frac{1}{2} h_{\alpha}^{\alpha} h_{\beta}^{\nu} R_{\nu}^{\beta}-\frac{1}{4} h_{\alpha, \nu}^{\beta} h_{\beta}^{\alpha, \nu} \\
& \left.+\frac{1}{4} h_{\alpha, \mu}^{\alpha} h_{\beta}^{\beta, \mu}-\frac{1}{2} h_{\alpha, \beta}^{\alpha} h_{\mu}^{\beta, \mu}+\frac{1}{2} h_{\beta}^{\nu, \alpha} h_{\alpha, \nu}^{\beta}\right] .
\end{aligned}
$$

Let us emphasize and clarify a number ofpoints:

(i) The original Lagrangian is invariant under gauge transformations of the fields $\bar{\phi}$ and $\bar{g}$. This implies invariance of this Lagrangian with respect to the gauge transformations (186), (187).

(ii) As a matter ofnotation, raising and lowering ofindices and covariant differentiation in eq. (190) is understood with respect to the background field $g_{\mu \nu}$ (seeeq.(184)).

(iii)By inspection we see that $\underline{\mathcal{L}}$ and $\underline{\underline{\mathcal{E}}}$, eqs. (189) and (190) are formally invariant under transformations whereby $\bar{h}, g, \phi$ and $\widetilde{\phi}$ transform as two tensors and scalars. We emphasize the difference with respect to the original gauge invariance. The transformations(186), (187) derive from eqs. (184) if we keep $g$ and $\widetilde{\phi}$ fixed, and assign tensor and scalar properties to $\bar{g}$ and $\phi$. That is the original gauge invariance of the theory. It will have to be broken by a gauge breaking term, and a ghost Lagrangian must be introduced. The Lagrangians $\underline{\mathcal{L}}$ and $\underline{\underline{\mathcal{E}}}$ are invariant if we assign transformation properties to $g, \widetilde{\phi}, h$ and $\phi$; for instance,

$$
h_{\mu \nu} \rightarrow h_{\mu \nu}+h_{\mu \alpha} \partial_{\nu} \eta^{\alpha}+h_{\alpha \nu} \partial_{\mu} \eta^{\alpha}+\eta^{\alpha} \partial_{\alpha} h_{\mu \nu} .
$$

This invariance needs not to be broken by a gauge breaking term because the classical functions $g$ and $\widetilde{\phi}$ are involved. The path integral

$$
\int \mathcal{D} h \mathcal{D} \phi \mathrm{e}^{i \int \underline{\underline{\mathcal{L}}}}
$$

is finite because $\mathcal{L}$ has no invariance with respect to variations of the quantum fields only. 
We must now also introduce a gauge breaking term in the original Lagrangian $\overline{\mathscr{L}}$ and also find the corresponding ghost Lagrangian. This will give rise to additions to $\underline{\mathcal{L}}$ and $\underline{\underline{\mathcal{L}}}$, and we must be careful that things are done such that the "new" invariance mentioned under (iii) remains true.

The following gauge breaking term does what we want:

$$
C_{\alpha}=\sqrt[4]{g}\left(h_{\mu, \nu}^{\nu}-\frac{1}{2} h_{\nu, \mu}^{\nu}-\phi \partial_{\mu} \widetilde{\phi}\right) t^{\mu \alpha},
$$

where $t^{\mu \alpha}$ is defined by

$$
t^{\mu \alpha} t^{\alpha \nu}=g^{\mu \nu} .
$$

We obtain

$$
\begin{aligned}
-\frac{1}{2} C_{\mu}^{2}= & -\frac{1}{2} \sqrt{g}\left(h_{\mu, \nu}^{\nu}-\frac{1}{2} h_{\nu, \mu}^{\nu}\right)\left(h_{, \alpha}^{\alpha \mu}-\frac{1}{2} h_{\alpha}^{\alpha, \mu}\right) \\
& +\sqrt{g}\left(h_{, \nu}^{\nu \mu}-\frac{1}{2} h_{\nu}^{\nu, \mu}\right) \phi \partial_{\mu} \tilde{\phi}-\frac{1}{2} \sqrt{g} \phi^{2} \partial_{\mu} \tilde{\phi} \partial_{\nu} \tilde{\phi} g^{\mu \nu} .
\end{aligned}
$$

The ghost Lagrangian is obtained by subjecting $C_{\alpha}$ from eq. (193) to the "original" gauge transformations(186), (187):

$$
C_{\nu} \rightarrow C_{\nu}+M_{\nu}+\mathrm{O}\left(\eta^{2}\right) .
$$

We find, omitting terms containing $h$ or $\phi$,

$$
M_{\nu}=\sqrt[4]{g}\left\{\eta_{, \mu \lambda}^{\lambda}+\eta_{\mu, \lambda}^{, \lambda}-\eta_{, \lambda \mu}^{\lambda}-\left(\partial_{\alpha} \widetilde{\phi}\right)\left(\partial_{\mu} \widetilde{\phi}\right) \eta^{\alpha}\right\} t^{\mu \nu} .
$$

Thus

$$
\mathcal{L}_{\text {ghost }}=\sqrt[4]{g} \eta_{\nu}^{*} M_{\nu},
$$

where now $\eta^{*}$ and $\eta$ represent the so-called Faddeev-Popov ghost field.

Without any consequence for the diagrams to be computed we may make the replacement

$$
\eta_{\nu}^{*} t^{\mu \nu} \rightarrow \sqrt[4]{g} \eta_{\nu}^{*} g^{\nu \mu} \equiv \sqrt[4]{g} \eta^{* \mu}
$$

provided $t$ is non-singular. We so fmd 


$$
\mathcal{L}_{\text {ghost }}=\sqrt{g} \eta^{* \mu}\left\{\eta_{\mu, \alpha}, \alpha-R_{\alpha \mu} \eta^{\alpha}-\left(\partial_{\alpha} \widetilde{\phi} \partial_{\mu} \widetilde{\phi}\right) \eta^{\alpha}\right\}
$$

This is invariant with respect to the "new" gauge transformations if we assign vector type transformation properties to the ghost field $\eta$.

Ofcourse, in actual calculations we may always use $\eta_{\mu}^{*}$ instead of $\eta^{* \mu}$, because the matrix $g$ involved is of no consequence.

It is perhaps worthwhile to mention here that we will be interested only in one-loop diagrams without external ghost lines. Therefore no term linear in the ghost (for example terms of the form $\eta h$ or $\eta \phi$ ) need to be retained. That is why terms containing $h$ or $\phi$ have been omitted.

Since $C$ is quadratic in the quantum fields $h, \phi$, only the Lagrangian $\underline{\underline{\mathcal{L}}}$ will get a contribution from the term $-\frac{1}{2} C^{2}$. One finds

$$
\begin{aligned}
& \underline{\underline{\mathcal{L}}}-\frac{1}{2} C_{\mu}^{2}=\sqrt{g}\left\{-\frac{1}{4} h_{\alpha, \nu}^{\beta} h_{\beta}^{\alpha, \nu}+\frac{1}{8} h_{\alpha, \nu}^{\alpha} h_{\beta}^{\beta, \nu}-\frac{1}{2} \partial_{\mu} \phi g^{\mu \nu} \partial_{\nu} \phi\right. \\
& \left.+\frac{1}{2} h_{\beta}^{\alpha} X_{\alpha \nu}^{\beta \mu} h_{\mu}^{\nu}+\phi Y_{\beta}^{\alpha} h_{\alpha}^{\beta}+\frac{1}{2} \phi Z \phi\right\} \\
& X_{\alpha \nu}^{\beta \mu}=2\left(-\frac{1}{2} \delta_{\nu}^{\beta} D^{\mu} \widetilde{\phi} D_{\alpha} \widetilde{\phi}+\frac{1}{4} \delta_{\alpha}^{\beta} D^{\mu} \widetilde{\phi} D_{\nu} \widetilde{\phi}-\frac{1}{16} \delta_{\alpha}^{\beta} \delta_{\nu}^{\mu} D_{\gamma} \widetilde{\phi} D^{\gamma} \widetilde{\phi}\right. \\
& +\frac{1}{8} \delta_{\nu}^{\beta} \delta_{\alpha}^{\mu} D_{\gamma} \widetilde{\phi} D^{\gamma} \widetilde{\phi}-\frac{1}{8} \delta_{\alpha}^{\beta} \delta_{\nu}^{\mu} R+\frac{1}{4} \delta_{\nu}^{\beta} \delta_{\alpha}^{\mu} R \\
& \left.-\frac{1}{2} \delta_{\nu}^{\beta} R_{\alpha}^{\mu}+\frac{1}{2} \delta_{\alpha}^{\beta} R_{\nu}^{\mu}+\frac{1}{2} R_{\alpha \nu}^{\beta \mu}\right), \\
& Y_{\beta}^{\alpha}=\frac{1}{2} \delta_{\beta}^{\alpha} D_{\nu} D^{\nu} \widetilde{\phi}-D_{\beta} D^{\alpha} \widetilde{\phi} \\
& Z=-D_{\mu} \widetilde{\phi} D^{\mu} \widetilde{\phi}
\end{aligned}
$$

\section{Evaluation of the divergencies}

The evaluation of the counter Lagrangian to (201) is straightforward but tedious. We must bring the Lagrangian in the form eq. (180), and after that we can use the general formula eq. (181). The fields $h_{\mu}^{\nu}$ and $\phi$ are to be identified with the $\phi^{i}$ of eq.(180); since in(180)the covariant derivative on the $\phi^{i}$ is treated as if working on a scalar field we must separate, in eq. (201), the terms $\partial_{\nu} h \partial_{\nu} h$ from the terms containing $\Gamma$. 
As a first step we apply the doubling trick to the Lagrangian (201). We get

$$
\begin{aligned}
\underline{\underline{\varrho}}_{\mathrm{d}}= & \sqrt{g}\left\{h_{\alpha \beta}^{*} P^{\alpha \beta \mu \nu} D_{\gamma} D^{\gamma} h_{\mu \nu}+\frac{1}{2} h_{\alpha \beta}^{*}\left(X^{\alpha \beta \mu \nu}+X^{\mu \nu \alpha \beta}\right) h_{\mu \nu}\right. \\
& \left.+\phi^{*} D_{\mu} D^{\mu} \phi+\phi^{*} Y^{\alpha \beta} h_{\alpha \beta}+h_{\alpha \beta}^{*} Y^{\alpha \beta} \phi+\phi^{*} Z \phi\right\}, \\
P^{\alpha \beta \mu \nu}= & \frac{1}{2} g^{\alpha \mu} g^{\beta \nu}-\frac{1}{4} g^{\alpha \beta} g^{\mu \nu}
\end{aligned}
$$

Let us consider the covariant derivatives. Let $T_{\alpha \beta \gamma}$... be a general tensor. We have

$$
D_{\mu} T_{\alpha \beta \gamma \ldots}=\partial_{\mu} T_{\alpha \beta \gamma \ldots}+N_{\mu \alpha \beta \gamma \ldots}^{\alpha^{\prime} \beta^{\prime} \gamma^{\prime} \ldots} T_{\alpha^{\prime} \beta^{\prime} \gamma^{\prime} \ldots},
$$

with

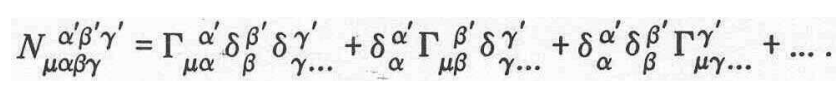

Further,

$$
\begin{aligned}
& D_{\nu} D_{\mu} T_{\alpha \beta \gamma \ldots}=\partial_{\nu} \partial_{\mu} T_{\alpha \beta \gamma \ldots}+\Gamma_{\nu \mu}^{\lambda} \partial_{\lambda} T_{\alpha \beta \gamma \ldots}+N_{\nu \alpha \beta \gamma \ldots}^{\alpha^{\prime} \beta^{\prime} \gamma^{\prime} \ldots \partial_{\mu}} T_{\alpha^{\prime} \beta^{\prime} \gamma^{\prime} \ldots}
\end{aligned}
$$

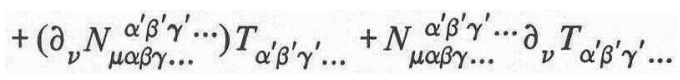

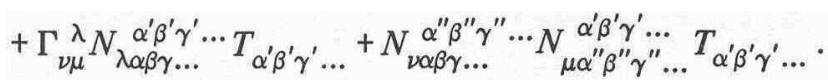

In short,

$$
D_{\nu} D_{\mu} T=\bar{D}_{\nu} \bar{D}_{\mu} T+N_{\nu} \bar{D}_{\mu} T+\left(\bar{D}_{\nu} N_{\mu}\right) T+N_{\mu} \bar{D}_{\nu} T+N_{\nu} N_{\mu} T .
$$

Here $\bar{D}$ is defined as the covariant derivative that "sees" only the indices $\mu$ and $v$ in eq. (206). From this we have for any tensor $T$,

$$
D_{\gamma} D^{\gamma} T=\bar{D}_{\gamma} \bar{D}^{\gamma} T+2 N^{\gamma} \bar{D}_{\gamma} T+\left(\bar{D}_{\gamma} N^{\gamma}+N_{\gamma} N^{\gamma}\right) T .
$$

Thus

$$
\begin{aligned}
\sqrt{g} h_{\alpha \beta}^{*} D_{\mu} D^{\mu} h_{\alpha \beta}= & \sqrt{g}\left\{h_{\alpha \beta}^{*} \bar{D}_{\mu} \bar{D}^{\mu} h_{\alpha \beta}\right. \\
& \left.+2 h_{\alpha \beta}^{*} N_{\alpha \beta}^{\mu \gamma \nu} \bar{D}_{\mu} h_{\gamma \nu}+h_{\alpha \beta}^{*} F_{\alpha \beta}^{\mu \nu} h_{\mu \nu}\right\},
\end{aligned}
$$


with

$$
F=\bar{D}_{\gamma} N^{\gamma}+N_{\gamma} N^{\gamma} .
$$

In the counter Lagrangian we will need $g_{\mu \nu}$,

$$
g_{\mu \nu}=\bar{D}_{\mu} N_{\nu}-\bar{D}_{\nu} N_{\mu}+N_{\mu} N_{\nu}-N_{\nu} N_{\mu} .
$$

The reader may note the great similarity between $\mathscr{G}_{\mu \nu}$ and the Riemann tensor $R_{\alpha \mu \nu}^{\beta}$ (see eq. (70)) in case $N$ is linear in the $\Gamma$. Indeed, one finds in the general case,

$$
\mathscr{G}_{\mu \nu \alpha \beta \gamma \ldots} \underset{\alpha^{\prime} \beta^{\prime} \gamma^{\prime} \ldots}{a}=R_{\alpha \mu \nu}^{\alpha^{\prime}} \delta_{\beta}^{\beta^{\prime}} \delta_{\gamma^{\prime} \ldots}^{\gamma^{\prime} \ldots}+\delta_{\alpha}^{\alpha^{\prime}} R_{\beta \mu \nu}^{\beta^{\prime}} \delta_{\gamma \ldots}^{\gamma^{\prime}}+\ldots .
$$

Here we have only two terms. We must be careful to symmetrize in $\alpha \beta$, and $\alpha^{\prime} \beta^{\prime}$. Thus,

$$
\Theta_{\mu \nu \alpha \beta}^{\alpha^{\prime} \beta^{\prime}}=\frac{1}{2}\left[R_{\alpha \mu \nu}^{\alpha^{\prime}} \delta_{\beta}^{\beta^{\prime}}+R_{\alpha \mu \nu}^{\beta^{\prime}} \delta_{\beta}^{\alpha^{\prime}}+R_{\beta \mu \nu}^{\alpha^{\prime}} \delta_{\alpha}^{\beta^{\prime}}+R_{\beta \mu \nu}^{\beta^{\prime}} \delta_{\alpha}^{\alpha^{\prime}}\right] .
$$

For this we find

$$
\operatorname{Tr}\left(g^{\mu \nu} g_{\mu \nu}\right)=g^{\mu^{\prime} \mu} g^{\nu^{\prime} \nu} 2 R_{\alpha^{\prime} \mu^{\prime} \nu^{\prime}}^{\alpha} \delta_{\beta}^{\beta^{\prime}} \frac{1}{2}[],
$$

where in between square brackets the symmetrized expression from eq. (213) must be used. Working this out gives

$$
\operatorname{Tr}\left(g^{\mu \nu} G_{\mu \nu}\right)=-6 R_{\alpha \beta \mu \nu} R^{\alpha \beta \mu \nu} .
$$

Due to the particular form of $F$, eqs. (209), (210), there are no more contributions from the covariant derivatives to the counter Lagrangian that indeed wiU involveonly $F-D_{\gamma} N^{\gamma}-N_{\gamma} N^{\gamma}=0$ (compareeq.(182)).

The rest of the calculation is now straightforward. Enumerating the fields $h_{\mu \nu}$ by a single index going from 1 to 10 , and assigning the scalar field $\phi$ to the index value 11 we find that the matrix $M$ takes the form

$$
M=\left[\begin{array}{ll}
P^{-1} \bar{X} & P^{-1} \bar{Y} \\
\bar{Y} & Z
\end{array}\right],
$$

where 


$$
\begin{aligned}
& \bar{X}^{\alpha \beta, \mu \nu}=\frac{1}{2} g^{\alpha \gamma} g^{\nu \pi} X_{\gamma \pi}^{\beta \mu}+\frac{1}{2} g^{\beta \gamma} g^{\mu \pi} X_{\gamma \pi}^{\alpha \nu}, \\
& \bar{Y}^{\alpha \beta}=g^{\alpha \nu} Y_{\nu}^{\beta} .
\end{aligned}
$$

The factors $P^{-1}$ arise from the substitution $h^{*} \rightarrow h^{*} P^{-1}$. We must now evaluate

$$
\operatorname{Tr}\left(M-\frac{1}{6} R\right)^{2},
$$

where $R$ multiplies the $11 \mathrm{X} 11$ unit matrix. The various contributions can be worked out,

$$
\begin{aligned}
& \operatorname{Tr}\left(P^{-1} \bar{X} P^{-1} \bar{X}\right)=2 R^{2}+6 R_{\alpha \beta} R^{\alpha \beta}+3\left(\partial_{\mu} \widetilde{\phi} g^{\mu \nu} \partial_{\nu} \widetilde{\phi}\right)^{2}, \\
& \operatorname{Tr}\left(-\frac{1}{3} P^{-1} X R+\frac{1}{36} R^{2}\right)=-\frac{31}{18} R^{2}, \\
& 2 \operatorname{Tr}\left(\bar{Y} P^{-1} \bar{Y}\right)=2\left(D_{\mu} D^{\mu} \widetilde{\phi}\right)^{2}+4 R^{\alpha \beta}\left(\partial_{\alpha} \widetilde{\phi} \partial_{\beta} \widetilde{\phi}\right), \\
& \operatorname{Tr}\left(Z-\frac{1}{6} R^{2}\right)=\frac{1}{3}\left(\partial_{\nu} \widetilde{\phi} g^{\mu \nu} \partial_{\mu} \widetilde{\phi}\right) R+\frac{1}{36} R^{2}+\left(\partial_{\mu} \widetilde{\phi} g^{\mu \nu} \partial_{\nu} \widetilde{\phi}\right)^{2} .
\end{aligned}
$$

The evaluation of the counter Lagrangian arising from the ghost loops is not difficult. The contribution due to the covariant derivatives is (we have now a single index field)

$$
\operatorname{Tr}\left(g^{\mu \nu} g_{\mu \nu}\right)_{\text {ghost }}=-R_{\alpha \beta \mu \nu} R^{\alpha \beta \mu \nu} .
$$

The minus sign is the well known minus sign for the ghost loops. From the rest of the ghost Lagrangian (200) one finds

$$
\begin{aligned}
\mathcal{L}_{\mathrm{c} \text { ghost }}= & -\frac{\sqrt{g}}{\epsilon}\left\{\frac{1}{6}\left(\partial_{\mu} \widetilde{\phi} g^{\mu \nu} \partial_{\nu} \widetilde{\phi}\right) R+\frac{1}{5} R^{2}+\frac{17}{30} R_{\alpha \beta} R^{\alpha \beta}\right. \\
& \left.+\frac{1}{12} R_{\mu \nu \alpha \beta} R^{\mu \nu \alpha \beta}+R^{\alpha \beta}\left(\partial_{\alpha} \widetilde{\phi} \partial_{\beta} \widetilde{\phi}\right)+\frac{1}{2}\left(\partial_{\mu} \widetilde{\phi} g^{\mu \nu} \partial_{\nu} \widetilde{\phi}\right)^{2}\right\} .
\end{aligned}
$$

Together with the previous result (divided by 2, to undo doubling), 


$$
\begin{aligned}
\mathcal{L}_{\mathrm{c}}= & \frac{\sqrt{g}}{\epsilon}\left\{\frac{9}{720} R^{2}+\frac{43}{120} R_{\alpha \beta} R^{\alpha \beta}+\frac{1}{2}\left(\partial_{\mu} \widetilde{\phi} g^{\mu \nu} \partial_{\nu} \widetilde{\phi}\right)^{2}\right. \\
& \left.-\frac{1}{12} R\left(\partial_{\mu} \widetilde{\phi} g^{\mu \nu} \partial_{\nu} \widetilde{\phi}\right)+2\left(D_{\mu} D^{\mu} \widetilde{\phi}\right)^{2}\right\} .
\end{aligned}
$$

Here we have used the identity

$$
R_{\mu \nu \alpha \beta} R^{\mu \nu \alpha \beta}=-R^{2}+4 R_{\alpha \beta} R^{\alpha \beta}+\text { total derivative . }
$$

This identity will be proven later.

\section{Equations of motion}

From eq. (189) we see that tadpoles are absent ifg and $\widetilde{\phi}$ obey the following equations of motion,

$$
\begin{aligned}
& D_{\mu} D^{\mu} \widetilde{\phi}=0, \\
& \left(-\frac{1}{2} R-\frac{1}{4} D_{\alpha} \widetilde{\phi} D^{\alpha} \widetilde{\phi}\right) \delta_{\nu}^{\mu}+R_{\nu}^{\mu}+\frac{1}{2} D_{\nu} \widetilde{\phi} D^{\mu} \widetilde{\phi}=0 .
\end{aligned}
$$

Taking the trace of eq. (225),

$$
R=-\frac{1}{2}\left(D_{\alpha} \widetilde{\phi} D^{\alpha} \widetilde{\phi}\right) .
$$

Substituting this back gives then the equivalent set

$$
D_{\mu} D^{\mu} \widetilde{\phi}=0, \quad R_{\mu \nu}=-\frac{1}{2}\left(D_{\mu} \widetilde{\phi}\right)\left(D_{\nu} \widetilde{\phi}\right), \quad R=-\frac{1}{2}\left(D_{\mu} \widetilde{\phi}\right)\left(D^{\mu} \widetilde{\phi}\right) .
$$

Using thsse equations in the counter Lagrangian (222) gives

$$
\mathscr{L}_{\mathrm{c}}=\frac{\sqrt{g}}{\epsilon} \frac{203}{80} R^{2} \quad \text { (scalar + gravity). }
$$

For pure gravity $R=0$, and

$$
\mathscr{L}_{\mathrm{c}}=0 \quad \text { (gravity). }
$$




\section{Riemann tensor identities}

Two completely antisymmetric four-tensors can be identified in four dimensions $\left(g=\operatorname{det} g_{\mu \nu}\right)$

$$
\begin{aligned}
& \eta^{\alpha \beta \mu \nu} \equiv \frac{1}{\sqrt{g}} \epsilon^{\alpha \beta \mu \nu}, \quad \eta_{\alpha \beta \mu \nu}=\sqrt{g} \epsilon_{\alpha \beta \mu \nu}, \\
& \epsilon_{\alpha \beta \mu \nu}=\epsilon^{\alpha \beta \mu \nu}=1 \quad \text { for } \quad \alpha, \beta, \mu, \nu=1,2,3,4 \\
&=\text { completely antisymmetric } .
\end{aligned}
$$

Under a gauge transformation $\xi^{\alpha}$ we have (see eq. (47))

$$
\sqrt{g} \rightarrow \sqrt{g}+\partial_{\alpha}\left\{\xi^{\alpha} \sqrt{g}\right\} \text {. }
$$

To see that the $\eta$ transforms indeed as tensors under a gauge transformation we write down an identity valid in four dimensions. Ifindices can take the values $1,2,3$ and 4 then obviously any object antisymmetric in five indices is zero. Thus consider

$$
\eta_{\mu \nu \alpha \beta} T_{\lambda \kappa \ldots}
$$

where $T_{\lambda \kappa \ldots}$ isanytensor. Thisisalreadyantisymmetricin $\mu, \nu, \alpha$ and $\beta$. If we antisymmetrize in the first five indices we have zero,

$$
\begin{gathered}
\eta_{\mu \nu \alpha \beta} T_{\lambda \kappa \ldots}-\eta_{\lambda \nu \alpha \beta} T_{\mu \kappa \ldots}-\eta_{\mu \lambda \alpha \beta} T_{\nu \kappa \ldots} \\
-\eta_{\mu \nu \lambda \beta} T_{\alpha \kappa}-\eta_{\mu \nu \alpha \lambda} T_{\beta \kappa}=0 .
\end{gathered}
$$

Let us now see how $\eta$ behaves under a gauge transformation. We have

$$
\begin{aligned}
& \eta_{\alpha \beta \mu \nu} \rightarrow \eta_{\alpha \beta \mu \nu}+\partial_{\lambda}\left\{\xi^{\lambda} \eta_{\alpha \beta \mu \nu}\right\} \\
& =\eta_{\alpha \beta \mu \nu}+\eta_{\alpha \beta \mu \nu} \partial_{\lambda} \xi^{\lambda}+\xi^{\lambda} \partial_{\lambda} \eta_{\alpha \beta \mu \nu} .
\end{aligned}
$$

Applying the antisymmetry identity to the second term

$$
\begin{aligned}
& \eta_{\alpha \beta \mu \nu} \rightarrow \eta_{\alpha \beta \mu \nu}+\eta_{\lambda \beta \mu \nu} \partial_{\alpha} \xi^{\lambda}+\eta_{\alpha \lambda \mu \nu} \partial_{\beta} \xi^{\lambda}+\eta_{\alpha \beta \lambda \nu} \partial_{\mu} \xi^{\lambda} \\
& +\eta_{\alpha \beta \mu \lambda} \partial_{\nu} \xi^{\lambda}+\xi^{\lambda} \partial_{\lambda} \eta_{\alpha \beta \mu \nu} .
\end{aligned}
$$


This is precisely the transformation law for a four-tensor. Similarly for $\eta^{\alpha \beta \mu \nu}$.

Let us now construct a scalar from two $\eta$ tensors and two Riemann tensors. Because of the various symmetry properties there is only one non-zero combination,

$$
\sqrt{g} R_{\lambda \sigma \alpha \beta} R_{\lambda^{\prime} \sigma^{\prime} \alpha^{\prime} \beta^{\prime}} \eta^{\lambda \sigma \alpha^{\prime} \beta^{\prime}} \eta^{\lambda^{\prime} \sigma^{\prime} \alpha \beta} \equiv \sqrt{g} R R \eta \eta .
$$

We will show that if $g_{\mu \nu} \rightarrow g_{\mu \nu}+\delta g_{\mu \nu}$ then this quantity changes by a total derivative. We have

$$
\begin{aligned}
& \sqrt{g} \rightarrow \sqrt{g}+\delta(\sqrt{g}), \\
& R \rightarrow R+\underline{R},
\end{aligned}
$$

where the first order variations $\delta(\sqrt{g})$ and $\underline{R}$ have been given before (see eqs. (84)-(97)). For our purposes we write somewhat more conveniently

$$
\begin{aligned}
& \delta(\sqrt{g})=\frac{1}{2} \sqrt{g} g^{\alpha \beta} \delta g_{\alpha \beta}, \\
& \underline{R}_{\nu \alpha \beta}^{\mu}=-D_{\beta} \underline{\Gamma}_{\nu \alpha}^{\mu}+D_{\alpha} \underline{\Gamma}_{\nu \beta}^{\mu}, \\
& \delta \eta^{\alpha \beta \mu \nu}=-\frac{1}{2} g^{\lambda \kappa} \delta g_{\lambda \kappa} \eta^{\alpha \beta \mu \nu},
\end{aligned}
$$

where $\underline{\Gamma}$ is the first order variation of $\Gamma$. This equation follows quite directly from the definition ofthe Riemann tensor, eq. (70). Furthermore,

$$
R_{\lambda \sigma \alpha \beta}=g_{\lambda \mu} R_{\sigma \alpha \beta}^{\mu} .
$$

From this

$$
\underline{R}_{\lambda \sigma \alpha \beta}=-g_{\lambda \mu} D_{\beta} \underline{\Gamma}_{\sigma \alpha}^{\mu}+g_{\lambda \mu} D_{\alpha} \underline{\Gamma}_{\sigma \beta}^{\mu}+\delta g_{\lambda \mu} g^{\mu \kappa} R_{\kappa \sigma \alpha \beta} .
$$

We find

$$
\begin{aligned}
& \delta\left(\sqrt{g} R_{\lambda \sigma \alpha \beta} R_{\lambda^{\prime} \sigma^{\prime} \alpha^{\prime} \beta^{\prime}} \eta^{\lambda \sigma \alpha^{\prime} \beta^{\prime}} \eta^{\lambda^{\prime} \sigma^{\prime} \alpha \beta}\right)=-\frac{1}{2} g^{\gamma \delta} \delta g_{\gamma \delta}(\sqrt{g} R R \eta \eta) \\
& \quad-4 \sqrt{g} g_{\lambda \mu}\left(D_{\beta} \Gamma_{\sigma \alpha}^{\mu}\right) R_{\lambda^{\prime} \sigma^{\prime} \alpha^{\prime} \beta^{\prime}} \eta^{\lambda \sigma \alpha^{\prime} \beta^{\prime}} \eta^{\lambda^{\prime} \sigma^{\prime} \alpha \beta} \\
& \quad+2 \sqrt{g} \delta g_{\lambda \mu} g^{\mu \kappa} R_{\kappa \sigma \alpha \beta} R_{\lambda^{\prime} \sigma^{\prime} \alpha^{\prime} \beta^{\prime}} \eta^{\lambda \sigma \alpha^{\prime} \beta^{\prime}} \eta^{\lambda^{\prime} \sigma^{\prime} \alpha \beta}
\end{aligned}
$$


If we had worked in $n$-dimensional space we would have $\eta$ tensors with $\eta$ indices, and the scalar combination of two $\eta$ 's with 4-index Riemann tensors would have needed $\frac{1}{2} n$ Riemann tensors. The coefficients of the last two terms would have been $-n$ and $\frac{1}{2} n$.

Let us now apply the antisymmetrization identity to the last term with respect to the indices $\kappa, \lambda, \sigma, \alpha^{\prime}$ and $\beta^{\prime}$. If we denote the last term of eq. (239) by $2 \sqrt{g} X$ we find the identity

$$
X=g^{\mu \lambda} \delta g_{\lambda \mu}(R R \eta \eta)-3 X,
$$

or

$$
X=\frac{1}{4} g^{\mu \lambda} \delta g_{\lambda \mu}(R R \eta \eta)
$$

In the $n$-dimensional case we would have obtained the coefficient $1 / n$. Inserting this identity we are left with

$$
\delta(\sqrt{g} R R \eta \eta)=-4 \sqrt{g} g_{\lambda \mu}\left(D_{\beta} \Gamma_{\sigma \alpha}^{\mu}\right) R_{\lambda^{\prime} \sigma^{\prime} \alpha^{\prime} \beta^{\prime}} \eta^{\lambda \sigma \alpha^{\prime} \beta^{\prime}} \eta^{\lambda^{\prime} \sigma^{\prime} \alpha \beta} .
$$

According to the Bianchi identities,

$$
D_{\beta} R_{\alpha^{\prime} \beta^{\prime} \lambda^{\prime} \sigma^{\prime}}+D_{\sigma^{\prime}} R_{\alpha^{\prime} \beta^{\prime} \beta \lambda^{\prime}}+D_{\lambda^{\prime}} R_{\alpha^{\prime} \beta^{\prime} \sigma^{\prime} \beta}=0 .
$$

Multiplying with $\eta^{\lambda^{\prime} \sigma^{\prime} \alpha \beta}$ we find three times the same thing, and consequently

$$
\left(D_{\beta} R_{\alpha^{\prime} \beta^{\prime} \lambda^{\prime} \sigma^{\prime}}\right) \eta^{\lambda^{\prime} \sigma^{\prime} \alpha \beta}=0 .
$$

Furthermore the covariant derivative of $\eta$ is easily seen to be zero,

$$
D_{\alpha} \eta^{\mu \nu \lambda \kappa}=0 \text {. }
$$

This follows basically from the identity

$$
\Gamma_{\alpha \kappa}^{\kappa}=\frac{1}{\sqrt{g}} \partial_{\alpha} \sqrt{g} .
$$

We therefore may rewrite

$$
\delta(\sqrt{g} R R \eta \eta)=\sqrt{g} D_{\beta}\left\{-4 g_{\lambda \mu} \Gamma_{\sigma \alpha}^{\mu} R_{\lambda^{\prime} \sigma^{\prime} \alpha^{\prime} \beta^{\prime}} \eta^{\lambda \sigma \alpha^{\prime} \beta^{\prime}} \eta^{\lambda^{\prime} \sigma^{\prime} \alpha \beta}\right\},
$$

since also the covariant derivative of $g_{\lambda \mu}$ is zero. Using 


$$
\sqrt{g} D_{\beta} X^{\beta}=\partial_{\beta}\left(\sqrt{g} X^{\beta}\right),
$$

we see indeed that the result is a total derivative.

As a last step we note that the product of two $\epsilon$ tensors can be expressed in products of $\delta$ functions,

$$
\eta^{\alpha \beta \mu \nu} \eta_{\alpha^{\prime} \beta^{\prime} \mu^{\prime} \nu^{\prime}}=\sum_{P}(-1)^{P} \delta_{\alpha^{\prime}}^{\alpha} \delta_{\beta^{\prime}}^{\beta} \delta_{\mu^{\prime}}^{\mu} \delta_{\nu^{\prime}}^{\nu} .
$$

The summation goes over all permutations of the indices $\alpha^{\prime}, \beta^{\prime}, \mu^{\prime}$ and $\nu^{\prime}$. There are 24 terms. Using this identity to work out the combination $R R \eta \eta$ as appearing in eq. (235) one obtains

$$
R R \eta \eta=4\left(R_{\mu \nu \alpha \beta} R^{\mu \nu \alpha \beta}-4 R_{\mu \nu} R^{\mu \nu}+R^{2}\right) .
$$

This expression is thus up to a constant a total derivative (in four dimensions) and we need to consider only terms of the form $R^{2}$ and $R_{\mu \nu} R^{\mu \nu}$ in the Lagrangian.

\section{Fermions and gravitation}

If one tries to include fermions into the theory of gravitation it turns out to be quite difficult. The reason for this is that there exists no spinor representation ofthe group oftransformations ofgeneral relativity. For this reason one is forced to introduce the Lorentz group once more, and treat the fermions as scalars with respect to general relativity. This is an ugly aspect of the theory of gravitation, but for the time being no alternative theory exists.

Let us now consider this problem explicitly. The Lagrangian for fermions not in interaction with gravitons is given by

$$
\mathscr{L}=-\bar{\psi}\left(\gamma^{\mu} \partial_{\mu}+m\right) \psi .
$$

To a given Lorentz transformation $L_{\mu \nu}$ corresponds a matrix $S$ in spinor space that acts on the $\psi$,

$$
\psi^{\prime}=S \psi,
$$

and the important properties of $S$ are (with $\bar{\psi}=\psi^{+} \gamma^{4}$ ) 


$$
S^{+} \gamma^{4}=\gamma^{4} S^{-1}, \quad S^{-1} \gamma^{\mu} S=L_{\mu \nu} \gamma^{\nu} .
$$

Thus the $S$ induce a Lorentz transformation of the $\gamma$ matrices.

Now the most straightforward generalization of eq. (248) would seem to be

$$
\mathcal{L}=\sqrt{g}\left\{-\bar{\psi}\left(\gamma^{\mu} D_{\mu}+m\right) \psi\right\} .
$$

This is acceptable if $\gamma^{\mu}$ transforms as a contravariant vector. The problem therefore arises: let there be given a gauge transformation characterized by the functions $\eta^{\alpha}$. Is it now possible to define a gauge transformation of the fields $\psi(x)$ in such a way that $\bar{\psi} \gamma^{\mu} \psi$ transforms as co- or contravariant vector? The answer to that is simply no.

For this reason we must now introduce the Lorentz group once more, ugly as it is. We introduce two kinds of indices, and the first kind indicates gauge transformation properties of general relativity, the other kind simply Lorentz transformation properties.

The central quantities in our treatment will be the so-called vierbein fields $e_{\mu}^{a}(x)$. A general gauge transformation is characterized by four functions $\eta^{\alpha}(x)$, and a Lorentz transformation is characterized by the six quantities $\lambda^{a b}(x)$, where $\lambda^{a b}=-\lambda^{b a}$. Thus $\delta^{a b}+\lambda^{a b}$ is simply an infinitesimal Lorentz transformation. The behaviour of the vierbein field $e_{\mu}^{a}$ under these transformations is given by

$$
e_{\mu}^{a} \rightarrow e_{\mu}^{a}+e_{\nu}^{a} \partial_{\mu} \eta^{\nu}+\eta^{\nu} \partial_{\nu} e_{\mu}^{a}+\lambda^{a b} e_{\mu}^{b} .
$$

Thus latin indices $a, b$, etc. indicate transformation with $\lambda$, and greek indices indicate transformation with $\eta$.

We must now construct covariant derivatives. Let there be given the fourvector $A^{a}(x)$. One has a gauge behaviour

$$
A^{a} \rightarrow A^{a}+\lambda^{a b} A^{b}+\eta^{\nu} \partial_{\nu} A^{a} .
$$

We now want to define the covariant derivative $D_{\mu}$ such that $D_{\mu} A^{a}$ transforms like the vierbein field. This can be done quite precisely like in the foregoing. Consider first $\partial_{\mu} A^{a}$

$$
\partial_{\mu} A^{a} \rightarrow \partial_{\mu} A^{a}+\lambda^{a b} \partial_{\mu} A^{b}+\partial_{\mu} \lambda^{a b} A^{b}+\partial_{\mu} \eta^{\nu} \partial_{\nu} A^{a}+\eta^{\nu} \partial_{\mu} \partial_{\nu} A^{a} .
$$


We want

$$
D_{\mu} A^{a} \rightarrow D_{\mu} A^{a}+\partial_{\mu} \eta^{\nu} D_{\nu} A^{a}+\eta^{\nu} \partial_{\nu} D_{\mu} A^{a}+\lambda^{a b} D_{\mu} A^{b}
$$

Comparison of(254) and (253) shows that we must try to find quantities $\omega_{\alpha}^{a b}$ such that under a gauge transformation

$$
\omega_{\alpha}^{a b} \rightarrow\left(\omega_{\alpha}^{a b}\right)_{\text {tensor }}-\partial_{\alpha} \lambda^{a b}
$$

This is provided by

$$
\omega_{\alpha}^{a b}=\frac{1}{2} e^{a \nu}\left(\partial_{\alpha} e_{\nu}^{b}-\partial_{\nu} e_{\alpha}^{b}\right)+\frac{1}{4} e^{a \rho} e^{b \sigma}\left(\partial_{\sigma} e_{\rho}^{c}-\partial_{\rho} e_{\sigma}^{c}\right) e_{\alpha}^{c}-(a \leftrightarrow b),
$$

where we used the definitions

$$
e_{\mu}^{a} e_{\nu}^{a} \equiv g_{\mu \nu}, \quad e^{a \nu} \equiv g^{\nu \sigma} e_{\sigma}^{a} .
$$

Thus the $g_{\mu \nu}$ are no more the basic quantities of the theory, but rather the $e_{\mu}^{a}$. The gravitational field is described by the difference between $e_{\mu}^{a}$ and unity,

$$
e_{\mu}^{a}=\delta_{\mu}^{a}+h_{a \mu}
$$

We have, to first order in the $h$,

$$
e_{\mu}^{a} e_{\nu}^{a}=\left(\delta_{\mu}^{a}+h_{a \mu}\right)\left(\delta_{\nu}^{a}+h_{a \nu}\right)=\delta_{\mu \nu}+\left(h_{\mu \nu}+h_{\nu \mu}\right) .
$$

The gravitational field that we had before is the symmetrical part of the vierbein field.

Let us now go back to eq. (256). To see that this has the correct behaviour one observes

$$
\begin{aligned}
& \partial_{\alpha} e_{\nu}^{b} \rightarrow\left(\partial_{\alpha} e_{\nu}^{b}\right)_{\text {tensor }}+e_{\mu}^{b} \partial_{\alpha} \partial_{\nu} \eta^{\mu}+\partial_{\alpha} \lambda^{b c} e_{\nu}^{c}, \\
& e^{a \nu}\left(\partial_{\alpha} e_{\nu}^{b}-\partial_{\nu} e_{\alpha}^{b}\right) \rightarrow()_{\text {tensor }}+\partial_{\alpha} \lambda^{b a}-e^{a \nu} e_{\alpha}^{c} \partial_{\nu} \lambda^{b c} \\
& e^{a \rho} e^{b \sigma}\left(\partial_{\sigma} e_{\rho}^{c}-\partial_{\rho} e_{\sigma}^{c}\right) e_{\alpha}^{c} . \\
& \rightarrow()_{\text {tensor }}+e^{b \sigma} \partial_{\sigma} \lambda^{c a} e_{\alpha}^{c}-e^{a \rho} \partial_{\rho} \lambda^{c b} e_{\alpha}^{c} .
\end{aligned}
$$


The rule for covariant differentiation is now clear. For example,

$$
D_{\mu} A_{\nu}^{a}=\partial_{\mu} A_{\nu}^{a}-\Gamma_{\mu \nu}^{\alpha} A_{\alpha}^{a}+\omega_{\mu}^{a b} A_{\nu}^{b},
$$

with the transformation properties that have been given before, eq. (255) and eq. (52).

Let us first list some equations concerning the vierbein field. We have

$$
g=\tilde{e} e \quad(\sim=\text { transpose }) .
$$

This is nothing but an abbreviated notation ofeq. (257). Now transposition and inversion commute,

$$
(\widetilde{e})^{-1}=\left(\widetilde{e^{-1}}\right),
$$

since

$$
e^{-1} e=1 \rightarrow \widetilde{e}\left(\tilde{e}^{-1}\right)=1 .
$$

Consider now

$$
e g^{-1} \widetilde{e} \quad\left(=e_{\mu}^{a} g^{\mu \nu} e_{\nu}^{b}\right) .
$$

We have

$$
e g^{-1} \widetilde{e}=e\left(e^{-1} \widetilde{e^{-1}}\right) \widetilde{e}=1,
$$

or

$$
e_{\alpha}^{a} e^{b \alpha}=\delta^{a b}
$$

Furthermore, the $\Gamma$ are related to the $\omega$. One has by inspection

$$
e_{\beta}^{b} \partial_{\nu} e_{\mu}^{b}+e_{\mu}^{c} \partial_{\nu} e_{\beta}^{c}=\partial_{\nu} g_{\mu \beta} .
$$

Using this one obtains (compare eq. (53))

$$
\begin{aligned}
e^{a \alpha} e_{\beta}^{b} \omega_{\mu}^{a b} & =\frac{1}{2} g^{\alpha \nu}\left(\partial_{\beta} g_{\mu \nu}-\partial_{\nu} g_{\mu \beta}+e_{\beta}^{b} \partial_{\mu} e_{\nu}^{b}-e_{\nu}^{a} \partial_{\mu} e_{\beta}^{a}\right) \\
& =\Gamma_{\beta \mu}^{\alpha}+\frac{1}{2} g^{\alpha \nu}\left(-\partial_{\mu} g_{\beta \nu}+e_{\beta}^{b} \partial_{\mu} e_{\nu}^{b}-e_{\nu}^{a} \partial_{\mu} e_{\beta}^{a}\right),
\end{aligned}
$$


or

$$
\Gamma_{\beta \mu}^{\alpha}=e^{a \alpha} e_{\beta}^{b} \omega_{\mu}^{a b}+e^{a \alpha} \partial_{\mu} e_{\beta}^{a} .
$$

From this one may derive that the covariant derivative of the vierbein field is zero,

$$
D_{\mu} e_{\beta}^{a}=\partial_{\mu} e_{\beta}^{a}-\Gamma_{\mu \beta}^{\alpha} e_{\alpha}^{a}+\omega_{\mu}^{a b} e_{\beta}^{b} .
$$

Using eqs. (267) and (264) we get

$$
D_{\mu} e_{\beta}^{a}=\partial_{\mu} e_{\beta}^{a}-e_{\beta}^{b} \omega_{\mu}^{a b}-\partial_{\mu} e_{\beta}^{a}+\omega_{\mu}^{a b} e_{\beta}^{b}=0 .
$$

\section{Fermionfields}

Under an infinitesimal Lorentz transformation $\lambda$ a fermion field is customarily supposed to transform according to

$$
\psi^{\prime} \rightarrow\left(1+\frac{1}{2} \sigma^{a b} \lambda^{a b}\right) \psi,
$$

with

$$
\sigma^{a b}=\frac{1}{4}\left(\gamma^{a} \gamma^{b}-\gamma^{b} \gamma^{a}\right)
$$

This is in fact the infinitesimal form of the transformations $S$ mentioned before. The only difference with the usual treatment is now that the $\lambda$ are spacetime dependent.

We must now define covariant differentiation of the $\psi$. We have evidently, from eq. (270),

$$
\partial_{\mu} \psi \rightarrow\left(1+\frac{1}{2} \sigma^{a b} \lambda^{a b}\right) \partial_{\mu} \psi+\frac{1}{2} \sigma^{a b} \partial_{\mu} \lambda^{a b} \psi \text {. }
$$

From this we infer that the correct definition of covariant derivative is

$$
D_{\mu} \psi=\left(\partial_{\mu}+\frac{1}{2} \sigma^{a b} \omega_{\mu}^{a b}\right) \psi
$$

Now the Lagrangian for fermions interacting with gravitons can be written

$$
\mathcal{L}_{\mathrm{f}}=e\left\{-\bar{\psi} \gamma^{a} e^{a \mu} D_{\mu} \psi-m \bar{\psi} \psi\right\},
$$


with $e=\operatorname{Det}(e)=\sqrt{\operatorname{Det} g}$. The gravitational Lagrangian is constructed from the $g_{\mu \nu}$ as before. The main difference is that we now have $e_{\mu \nu}+e_{\nu \mu}$ instead of $h_{\mu \nu}$, which introduces some extra symmetrization in a number of places. As an interesting trick we make the substitution

$$
\bar{\psi} \rightarrow D_{\nu} \bar{\psi} \gamma^{b} e^{b \nu} .
$$

This gives (we ignore the mass term here)

$$
\mathcal{L}_{\mathrm{f}}=e\left\{-D_{\nu} \bar{\psi} \gamma^{a} \gamma^{b} e^{b v} e^{a \mu} D_{\mu} \psi\right\} .
$$

This is symmetric in $\mu$ and $v$ : interchange of $\mu$ and $v$ can be obtained via partial differentiation, using that $D e=0$. Therefore we get symmetry in $a$ and $b$, and we may substitute

$$
\gamma^{a} \gamma^{b} \rightarrow \frac{1}{2}\left(\gamma^{a} \gamma^{b}+\gamma^{b} \gamma^{a}\right)=\delta^{a b} .
$$

We so find

$$
\mathcal{L}_{\mathrm{f}}=e\left[-D_{\nu} \bar{\psi} g^{\mu \nu} D_{\mu} \psi\right] .
$$

\section{Further invariants}

Consider now the tensor $R_{\mu \nu}^{a b}$ defined by

$$
\left(D_{\mu} D_{\nu}-D_{\nu} D_{\mu}\right) \psi \equiv \frac{1}{2} R_{\mu \nu}^{a b} \sigma^{a b} \psi .
$$

One has

$$
\begin{aligned}
& D_{\nu} \psi=\partial_{\nu} \psi+\frac{1}{2} \omega_{\nu}^{a b} \sigma^{a b} \psi \\
& D_{\mu} D_{\nu} \psi=\partial_{\mu} D_{\nu} \psi-\Gamma_{\mu \nu}^{\alpha} D_{\alpha} \psi+\frac{1}{2} \omega_{\mu}^{c d} \sigma^{c d} D_{\nu} \psi \\
&=\partial_{\mu} \partial_{\nu} \psi+\frac{1}{2} \partial_{\mu}\left(\omega_{\nu}^{a b} \sigma^{a b}\right) \psi+\frac{1}{2} \omega_{\nu}^{a b} \sigma^{a b} \partial_{\mu} \psi \\
&-\Gamma_{\mu \nu}^{\alpha} D_{\alpha} \psi+\frac{1}{2} \omega_{\mu}^{c d} \sigma^{c d} \partial_{\nu} \psi+\frac{1}{4} \omega_{\mu}^{c d} \omega_{\nu}^{a b} \sigma^{c d} \sigma^{a b} \psi
\end{aligned}
$$


Using equations such as

$$
\left[\sigma^{c d}, \sigma^{a b}\right]=\delta^{b d} \sigma^{a c}+\delta^{a d} \sigma^{c b}+\delta^{b c} \sigma^{d a}+\delta^{a c} \sigma^{b d},
$$

that follow from the definition eq. (271) one finds

$$
R_{\mu \nu}^{a b}=\partial_{\mu} \omega_{\nu}^{a b}-\partial_{\nu} \omega_{\mu}^{a b}+\omega_{\mu}^{a c} \omega_{\nu}^{c b}-\omega_{\nu}^{a c} \omega_{\mu}^{c b},
$$

which indeed is quite analogous to eq. (70). Obviously $R^{a b}$ behaves as a tensor, which can also be deduced directly, if we first rewrite

$$
R_{\mu \nu}^{a b}=D_{\mu} \omega_{\nu}^{a b}-D_{\nu} \omega_{\mu}^{a b}-\omega_{\mu}^{a c} \omega_{\nu}^{c b}+\omega_{\nu}^{a c} \omega_{\mu}^{c b},
$$

and then use the transformation properties of $\omega$, eq. (255).

The vierbein field can be used to "transform" a latin index into a greek index and vice versa. For instance

$$
e^{a \alpha} e_{\beta}^{b} R_{\mu \nu}^{a b}=R_{\beta \mu \nu}^{\alpha}
$$

This may be seen as follows:

$$
\begin{aligned}
e^{a \alpha} e_{\beta}^{b} R_{\mu \nu}^{a b} & =e^{a \alpha} e_{\beta}^{b}\left(D_{\mu} \omega_{\nu}^{a b}-D_{\nu} \omega_{\mu}^{a b}\right)-e^{a \alpha} e_{\beta}^{b}\left(\omega_{\mu}^{a c} \omega_{\nu}^{c b}-\omega_{\nu}^{a c} \omega_{\mu}^{c b}\right) \\
& =D_{\mu} \Gamma_{\beta \nu}^{\alpha}-D_{\nu} \Gamma_{\beta \mu}^{\alpha}-D_{\mu}\left(e^{a \alpha} \partial_{\nu} e_{\beta}^{a}\right)+D_{\nu}\left(e^{a \alpha} \partial_{\mu} e_{\beta}^{a}\right) \\
& -e^{a \alpha} \omega_{\mu}^{a c} e_{\beta}^{b} \omega_{\nu}^{c b}+e^{a \alpha} \omega_{\nu}^{a c} e_{\beta}^{b} \omega_{\mu}^{c b}
\end{aligned}
$$

where we used the fact that the covariant derivative of the $e$ is zero. We leave it to the reader to work out further eq. (282).

\section{References}

The following very incomplete list gives a number of references that are rather closely related to the type of treatment exposed in these notes.

\section{General}

[1] R.P. Feynman, Lectures on gravitation, Cal. Tech. Lecture Notes 1962-63.

[2] B. De Witt, in Relativity, groups and topology, Les Houches 1963.

[3] G. 't Hooft and M. Veltman, Diagrammar, CERN Yellow report 73-9 (1973). 


\section{Graviton propagator}

[4] H. Van Dam and M. Veltman, Nucl. Phys. B22 (1970) 397.

[5] P. Van Nieuwenhuizen, Phys. Rev. D7 (1973) 2300. This paper contains further references to the subject.

\section{Divergencies}

[6] G. 't Hooft and M. Veltman, Ann. Inst. Henri Poincare 20 (1974) 69.

[7] S. Deser and P. Van Nieuwenhuizen, Phys. Rev. Letters 32 (1974) 245; Phys. Rev. D10(1974)401,411.

[8] S. Deser, H.S. Tsao and P. Van Nieuwenhuizen, Phys. Letters 50B (1974) 491; Phys. Rev. D10 (1974) 3337.

[9] J.M. Cornwall, D. Levin and G. Tiktopoulos, Phys. Rev. Letters 30 (1973) 1268; 31 (1973)572(E);Phys. Rev.D10(1974) 1145; andarecentpreprint.

[10] D. Freedman, I. Muzinich and E. Weinberg, Ann. of Phys. 87 (1974) 95.

[11] R. Kallosh, Phys. Letters 55B (1975) 321.

[12] S. Deser, Grisaru and P. Van Nieuwenhuizen, Brandeis Univ. Preprint 1975.

[13] D. Freedman and E. Weinberg, Ann. of Phys. 87 (1974) 354.

[14] D. Freedman and So-Young Pi, Ann. of Phys. 91 (1975) 442.

[15] J.G. Taylor and M. Nouri-Maghodom, King's College preprint 1975.

Finite effects of quantum gravity and other related subjects

[16] F. Berends and R. Gastmans, Phys. Letters 55B (1975) 311;Nucl. Phys. B88 (1975) 99. These papers consider quantum gravity interplay with quantum electrodynamics.

[17] F. Englert, E. Gunzig, C. Truffin and P. Windey, Faculte des Sciences, Univ. Libre de Bruxelles, Phys. Letters 57B (1975) 73.

[18] D.M. Capper and M. Duff, Phys. Letters 53A (1975) 361; Nuovo Cimento 23A (1974) 173.

[19] E. Fradkin and G. Vilkovisky, Phys. Rev. D8 (1973) 4241. This and the previous papers contain many useful references.

[20] L. Faddeev and V. Popov, Sov. Phys. Usp. 16 (1974) 777. 


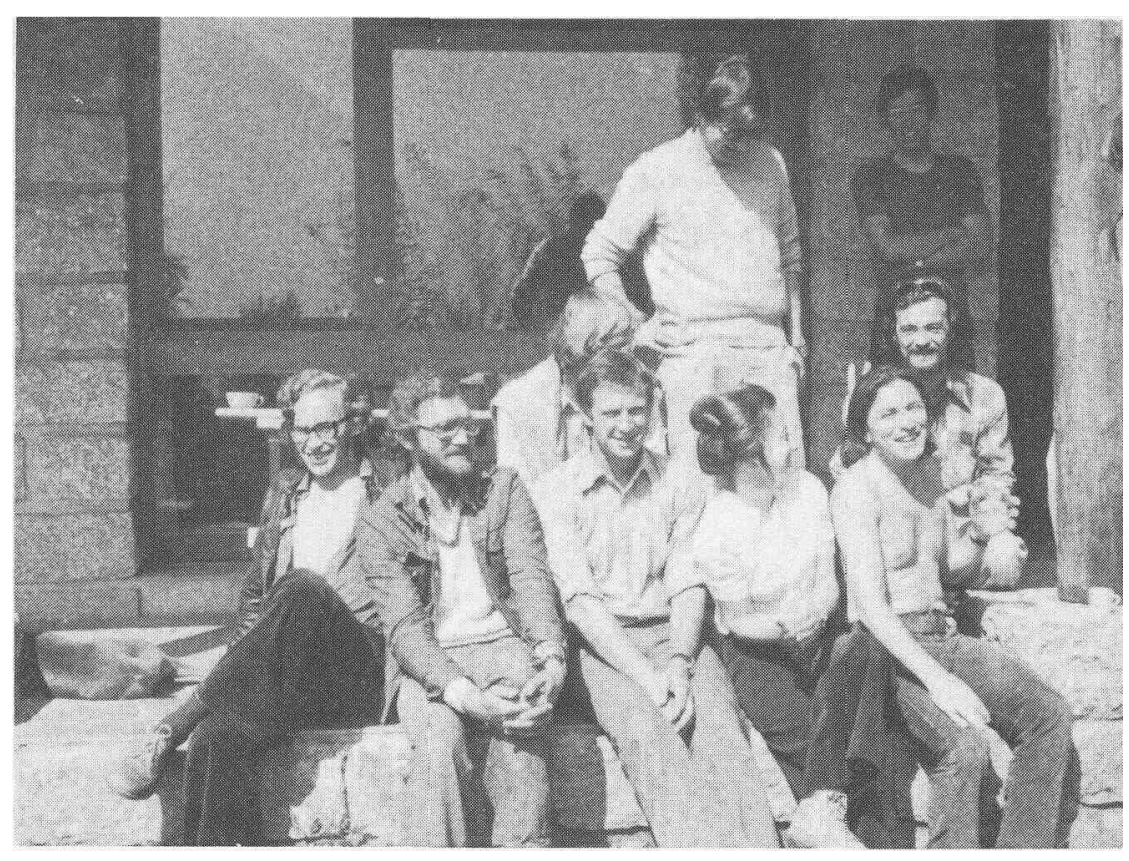

